\title{
COMMENTED DISTRIBUTIONAL LIST OF THE REPTILES OF MAURITANIA (WEST AFRICA)
}

\author{
J. M. Padial ${ }^{1}$
}

\begin{abstract}
This is the first comprehensive review of the reptiles of Mauritania. It includes distributional information and comments. Mauritania harbors 86 species of reptiles belonging to 21 families. Among these families, Colubridae and Lacertidae are the most diverse, with 14 and 13 species respectively. Other families, such as Agamidae, Gekkonidae, Scincidae or Viperidae are also well represented. Among the 80 continental species, $47.5 \%$ are Saharan, 33.8\% Afrotropical, 16.2\% Sahelian and 2.5\% Mediterranean. The marine turtles form another important group, with six species. Eight species are excluded from the country list because of old identification errors, there is not enough evidence of their presence or due to changes in political borders. Among the species expected to occur in Mauritania, at least nine may occur in Saharan environments, 13 in the Sahel savannah and two may have been introduced.
\end{abstract}

Key words: Distribution, checklist, Mauritania, Africa, Reptilia, Sahara, Sahel.

\section{RESUMEN}

\section{Lista comentada de los reptiles de Mauritania (oeste de África)}

Esta es la primera lista de reptiles comentada con datos de distribución para la República Islámica de Mauritania. La fauna de reptiles de Mauritania se compone de 86 especies pertenecientes a 21 familias. Entres ellas, Colubridae y Lacertidae son las más diversas, con 14 y 13 especies respectivamente. Otras familias como Agamidae, Gekkonidae, Scincidae y Viperidae también son representativas. Entre las 80 especies continentales el $47.5 \%$ son de distribución sahariana, el $33.8 \%$ afrotropical, el $16.2 \%$ sahelianas y el $2.5 \%$ mediterráneas. Las tortugas marinas, con seis especies, es otro grupo importante. Ocho especies no han sido incluidas en la lista debido a que se trataba de viejos errores de identificación, por carecer de suficiente evidencia o debido a la redistribución de las viejas fronteras políticas. Entre las especies potencialmente presentes, al menos nueve de ellas se esperan para las zonas saharianas, trece para las sabanas del Sahel, y dos podrían haber sido introducidas.

Palabras clave: Distribución, lista comentada, Mauritania, África, Reptilia, Sáhara, Sahel.

\footnotetext{
* Department of Biodiversity and Evolutionary Biology, Museo Nacional de Ciencias Naturales-CSIC. C/ José Gutierrez
} Abascal 2, 28006, Madrid, Spain. E-mail: jmpadial@yahoo.com 


\section{Introduction}

Mauritania is one of the herpetologically least known countries of Africa. During the last twenty years, Morocco, West Sahara and even Mali have received considerable attention from herpetologists (Bons \& Geniez, 1996; Schleich et al., 1996; Geniez et al., 2004). Mauritania was almost forgotten by naturalists after the French colonization and establishment of the current political borders. Most natural history information was produced before the sixties by French naturalists who collected from mainly coastal areas and populated places. Some expeditions took place during the beginning of the last century in what we know today as the Islamic Republic of Mauritania. Naturalists such as M. Chudeau, M. Audan, G. Bouet, T. Monod, and M. Murat collected most of the specimens that were subsequently studied by Pellegrin, Chabanaud, Angel, Villiers, etc. The first author that reported some collections from Mauritania was Moquard (1895), who wrote about the reptiles of Cap Blanc, in the former French West Africa. Subsequently, Pellegrin (1910) studied the specimens collected during an expedition directed by M. Gruve in 1908 between Saint Louis and Cap Blanc, and reported eight species. Chabanaud (1916, 1917) studied Audan's specimens collected during 1909, 1912 and 1913. Also Boulenger (1919) contributed some information for the region, based on material deposited in the British Museum. However, he referred to "Mauritania" in a broad sense, probably including parts of West Sahara. He also studied some specimens collected by Audan in the Sahelian Trarza region. Böttger (1921) commented that the distribution of some reptiles found by him in the former Spanish colony of "Río de Oro", reached the Mauritanian Cap Blanc and further corrected one observation of Pellegrin about the coastal Stenodactylus species. Angel (1938, 1939) studied the material collected by $M$. Murat during the expedition "D'études de la biologie des Acridiens", directed by M. Zolotarevsky in 1936 and 1937 along the central and northwestern part of the country. He described the subspecies Eryx muelleri subniger and reported information for another twelve species.

After these early contributions, Mauritania received more attention, mainly through the works of Villiers (1950, 1951, 1954, 1956, 1975), who commented and described the distribution and/or some aspects of the natural history of at least 23 snakes from Mauritania. His snake checklist for
Mauritania (Villiers, 1950) was the obligatory reference for subsequent revisions of the Mauritanian herpetofauna. Moreover, Dekeyser \& Villiers (1956) completed the most thorough ecological and faunistical study performed to date in Mauritania. They analyzed the distribution by habitats and microhabitats of plant and animal species in the central area of Adrar, and reported the presence of many reptiles, some of which are very difficult to detect even today. After this relatively intense activity before Mauritania's independence in 1960, no other herpetological survey took place in the country, except those of Maigret (1975) and Le Toquin et al., (1980) on marine turtles. Nevertheless, more general taxonomic works included specimens from Mauritania (i.e Grandison, 1956, 1961). Already in the late seventies and eighties, the interest in African herpetology increased. Taxonomic revisions (Arnold \& Levinton, 1977; Joger, 1980, 1981, 1984; Salvador, 1982; Schätti, 1986; Hahn \& Wallach, 1998) and biogeographical or distributional analyses (i.e. Maigret, 1983; Lambert, 1984; Hughes, 1983), included information on Mauritania and, furthermore, included specimens collected during earlier expeditions. These efforts led to the description of new species and contributed to the unravelling and/or identification of several taxonomic problems. Welch's (1982) list and Leberre's (1989) popular book were the more comprehensive lists published on Mauritanian herpetofauna at this time. But other studies in adjacent areas, for example Senegal (Böhme, 1978; Cissé \& Karns, 1979) or Morocco (Bons \& Geniez, 1996), served to illustrate how incomplete the herpetological knowledge of Mauritania was. In more recent times we have seen a steady increase in herpetological research in Mauritania. This was probably favoured by the rapid increase of knowledge of other West African countries (i.e. Bons \& Geniez, 1996; Joger \& Lambert, 1996; Schleich et al., 1996), and political stability. Thus, during the nineties and early $21^{\text {st }}$ Century, many naturalists contributed to the knowledge of the Mauritanian herpetofauna. Bons \& Geniez (1996) provided some information for northern Mauritania; while Ineich (1997) compiled the most complete reptile list to date (33 species), as a result of his research along the coastal area; Arvy et al., (1996), Arvy \& Dia (1997) and Arvy et al., (1997) increased our knowledge of turtles; many papers contributed with new discoveries and additional distributional information (Hasi et al., 1997; Lambert \& Mullie, 1998; Donaire et al., 2000; Böhme, 2000; Böhme 
Fig. 1.- Map of the Islamic Republic of Mauritania showing main administrative divisions (Wilayas).

Fig. 1.- Mapa de la República Islámica de Mauritania y sus principales divisiones administrativas (Wilayas).

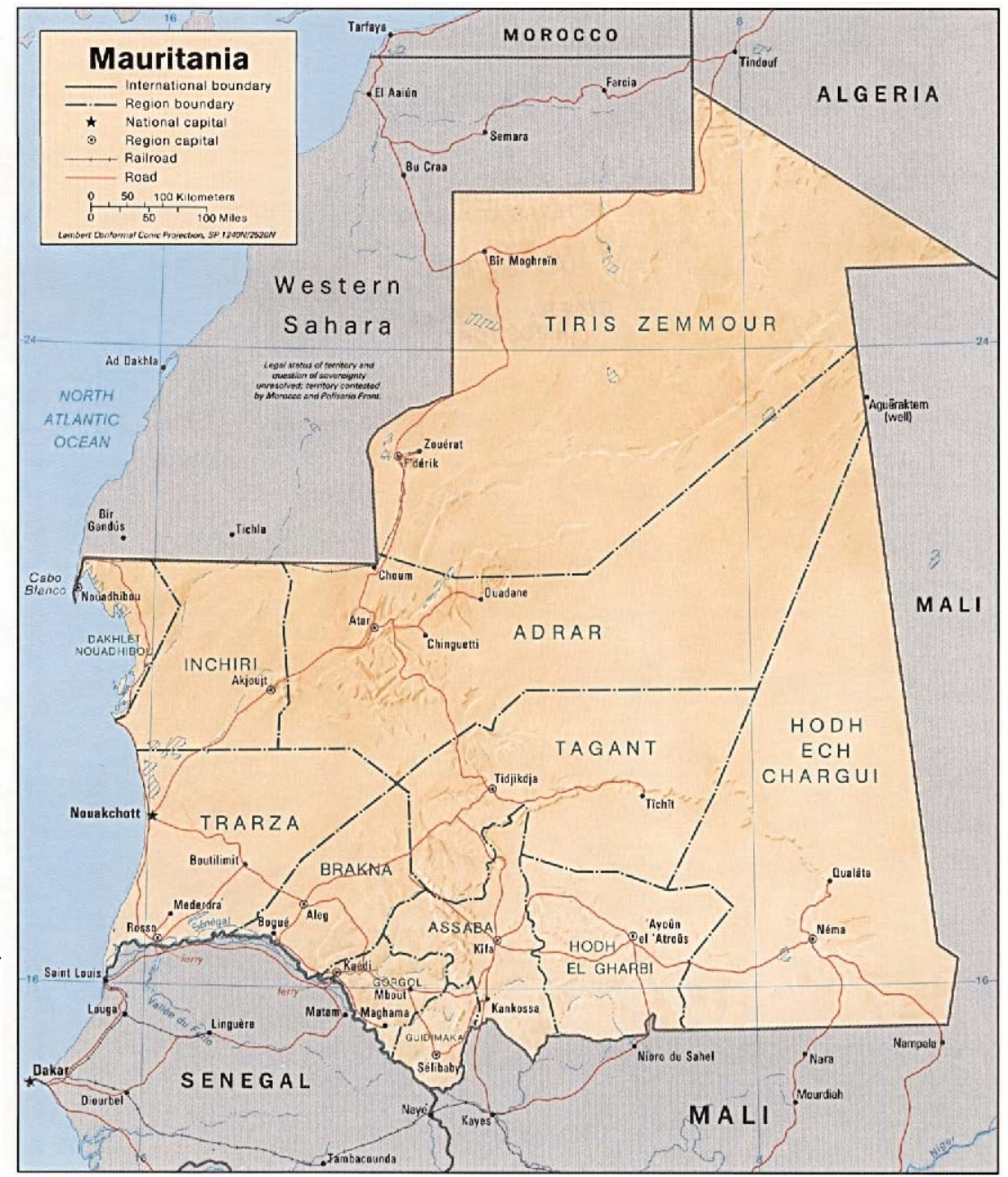

et al., 2001; Shine et al., 2001; Padial et al., 2002; Padial, 2003; Geniez \& Luch, 2004; Aymerich et al., 2004). Moreover, some species were described from neighboring countries (Geniez \& Foucart, 1995; Joger \& Lambert, 1996; Mateo et al., 1998) and subsequently reported from Mauritania. Other species were described exclusively for Mauritania (Wallach, 2002; Padial, 2005; Geniez \& Arnold, 2006). In addition, the systematics of some complex groups was analyzed (Mateo et al., 1998; Wilms \& Böhme, 2001; Crochet et al., 2003). Finally Geniez et al., (2000; 2004) and Chippaux (2001) added more herpetological information for Mauritania. Geniez et al.'s (2004) review was the most comprehensive herpetofaunistic list for northern Mauritania to date.

National species lists are of high relevance for conservation policy and scientific research (Padial \& De la Riva, 2006). Biodiversity is managed at the political level, and international founding is prioritized for endangered species, biodiversity hotspots, areas of endemism, etc. In this context, the Mauritanian herpetofauna lies outside the scope of serious attention, since there is no scientific list at all [the first list for amphibians was recently published by Padial \& De la Riva (2004)]. The only available sources for general information on Mauritanian reptile diversity are on the web: the EMBL Reptile Database (2006) and the WCMC (2006). But they contain some outdated information and old mistakes. Whereas some countries around Mauritania have already been herpetofaunistically analyzed (see above), Mauritania urgently needs a reptilian species list.

My recent fieldwork in Mauritania and efforts in compiling and analyzing all available herpetological information from the country have led to this preliminary checklist. I have removed some erro- 
neous records and also included taxonomic changes, new records, and a thorough literature revision. I also include a preliminary list of some species expected to occur. In fact, the reptile diversity in the country has been seriously underestimated and many areas need to be surveyed. Mauritania could play a very relevant role in conservation strategies and biogeographical analyses of the West African herpetofauna. I hope this list may facilitate all future observations on Mauritanian reptiles and provide a baseline for other herpetological studies and more general biodiversity research in the country.

\section{Methods}

Fieldwork was performed between April and August 2002. Specimens were collected only when they belonged to species with taxonomic problems or when they were not previously reported for the country. Common and easily identifiable species were collected by hand, photographed and released. Some specimens were found dead along the road or killed by local people. Specimens were sacrificed with injection of nicotine, fixed in $10 \%$ formalin, and preserved in ethanol $70^{\circ}$. They are deposited either in the Centro de Estudios Tropicales, Sevilla, Spain (CET) or the Museo Nacional de Ciencias Naturales, Madrid, Spain (MNCN). Tissue samples were deposited in the British Museum of Natural History (London). I tried to review and contrast all published literature about the herpetofauna of Mauritania. Nevertheless, it is possible that some localities, records or minor references may have been overlooked. In the text, scientific names are followed by the distribution and/or comments about the species. Localities are ordered by "wilayas" (main administrative divisions of the country, see Fig. 1). The wilayas are underlined. Localities are ordered alphabetically, and are followed by their respective references or the initials JMP in cases where I found the species in the respective locality. The Appendix compiles the coordinates of the localities extracted from the literature or visited by the author. Nevertheless, for some localities the coordinates could not be found. For general toponymy I followed the Carte Générale of Mauritania (1:2500000) (IGN, 1993). Some names may change when translated from French to Hassaniya or viceversa, hence, spelling of some localities can vary in different cartographies.

\section{Results}

ANNOTATED LIST

\section{REPTILIA \\ SAURIA \\ AGAMIDAE}

\section{Agama agama Linnaeus, 1758}

Distribution: Adrar: Atar (Joger, 1979), Chinguetti (Dekeyser \& Villiers, 1956); Guidimaka: Bouli (Padial, 2005); Foulania (Padial, 2005); Zoueina (Padial, 2005);

Comments: Joger (1979) considered the Atar population of A. agama as a recent colonization. Some records of this species in desert habitats may correspond to $A$. impalearis. Grandison (1956) cited A. agama for Mauritania without locality.

Agama boueti Chabanaud, 1917

Distribution: Assaba: Bougari (Nickel, 2003); Metraucha (Nickel, 2003); Hodh Ech Chargui: Mahmûdé Lake (Nickel, 2003); Hodh El Gharbi: Ayoûn El Atroûs (Böhme et al., 2001; Padial, 2005); Trarza: Chott Boul (Ineich, 1997); Dar es Salam (Ineich, 1997); Muri (JMP); Northern from Rosso (Böhme et al., 2001).

Comments: Le Berre (1989) already cited this species for the country. Joger (1979) noted that this species inhabits southern Mauritania. Indeed, it is common in sandy savannas from southern Mauritania and also from the southern coastal area. See comments on $A$. castroviejoi.

\section{Agama boulengeri Lataste, 1886}

Distribution: Adrar: Aggui (Geniez et al., 2004); Ben Amira (Padial, 2005); Chinguetti (Pleguezuelos et al., 2005); Guelta Molomhar (JMP), Guelta Oumm Lebare (Padial, 2005); Guelta Hamdoun (Dekeyser \& Villiers, 1956); Iriji (Dekeyser \& Villiers, 1956); Kanoal, Oued Séguelil (Dekeyser \& Villiers, 1956); Oued Choûm (Padial, 2005); Terjît (Padial, 2005); Zerga mountains, about $34 \mathrm{~km}$ WSW of Chinguetti (Geniez \& Arnold, 2006); $44 \mathrm{~km}$ from Chinguetti on the road to Adrar, $19 \mathrm{~km}$ before the Nouatil Pass (Geniez \& Arnold, 2006); Assaba: Aïn El Ghaire (Lambert \& Mullié, 1998); Bou Bleî'n̂ne (Padial, 2005); Bougari (Padial, 2005); Guelta Oumm Lebare (Padial, 2005); Metraucha (Nickel, 2003; Padial, 2005); near Louths (Lambert \& Mullié, 1998); Brakna: Achram (Lambert \& Mullié, 1998); Gorgol: M'bout (JMP); Guidimaka: Sélibabi (JMP); Hodh Ech Chargui: Between Timbedgha and Ayoûn El Atroûs 
(Böhme et al., 2001; Padial, 2005); Hodh El Gharbi: Ayoûn El Atroûs (Padial, 2005); Oumm El Khez (JMP); Tintâne (JMP); Tagant: Guelta Fanar (JMP); Guelta Matmata (Padial, 2005); between Lekhcheb and Tîchît (Padial, 2005); Tidjikja (JMP).

COMMENTS: voucher specimens from Mauritania are in BM and museum IFAN in Dakar (Joger, 1979). The specimens of Oued Choum reported by Padial (2005) represent the northernmost record for this species.

\section{Agama castroviejoi Padial, 2005}

Distribution: Adrar plateau, road from Atar to Tidjikja (Padial, 2005); road from Atar to Chinguetti via Ebnou Pass (Brito, 2003); $44 \mathrm{~km}$ from Chinguetti on the road to Adrar, $19 \mathrm{~km}$ before the Nouatil Pass (Geniez \& Arnold, 2006).

COMMENTS: I assign the recent record of Agama boueti for the Adrar, near the type locality of $A$. castroviejoi, (Brito, 2003) to A. castroviejoi, a recently described species very similar to $A$. boueti and endemic to this mountain range.

\section{Agama impalearis Böttger, 1874}

Distribution: Adrar: Amogjar pass, c. $50 \mathrm{~km}$ East of Atar (Brito, 2003); Road from Atar to Chinguetti (JMP).

CoMmENTs: Chabanaud (1917) cited Agama colonorum for "Mauritanie saharienne" based on a single individual collected by Audan. Despite Le Berre's (1989) distribution map for A. impalearis, where this species appears to occur widely across the country, no concrete records were known up to then. Joger (1979) noted that this species did not occur in Mauritania and that the nearest record was Sequia el Hamra (West Sahara). Nevertheless, Brito (2003) recently confirmed the presence of this species in the Adrar. I also observed some gregarious specimens perched on rocks in the Adrar plateau between Adrar and Chinguetti. These may correspond to the Southern form of $A$. impalearis, but no specimen could be captured for confirmation.

\section{Trapelus mutabilis (Merrem, 1820)}

Distribution: Adrar: El Beyyed (JMP); Kanoal, Oued Séguelil (Dekeyser \& Villiers, 1956); ca. $50 \mathrm{~km}$ south of Choum, road Choum-Atar (Brito, 2003); Ouadâne area (Geniez et al., 2004); Terjît (JMP); Dakhlet Nouâdhibou: Banc d'Arguin (Padial et al., 2002); between Tintan and Akjoujt (Pellegrin, 1910); Inâl (Geniez et al., 2004); Tintan (Pellegrin, 1910); Alsaz (Pellegrin, 1910), Manata (Pellegrin, 1910); Boû Lanouâr; c. $50 \mathrm{~km}$ east of Boû Lanouâr, track
Nouadhibou-Choum (Brito, 2003); Oued Khatt Atoui (Angel, 1938); Tasiast (Angel, 1938, 1939); Inchiri: Agneïtîr (Ineich, 1997); Azeffâl (Ineich, 1997); Bennichchâb (JMP); between Tiouilit and Bennichchâb (Pleguezuelos et al., 2005); Tiris Zemmour: near Toûâjil (JMP); between Choum and Toûâjil (Geniez et al., 2004).

\section{Uromastyx dispar Rüppel, 1827}

DisTRIBUTION: Adrar: Ain Savra (JMP); Amogjar pass, ca $50 \mathrm{~km}$ E of Atar (Brito, 2003); Atar (terra típica) (Böhme et al., 2001; Wilms \& Böhme, 2001); Atar, 65 km S (JMP); Ar Bou M'rait (Wilms \& Böhme, 2001); $53 \mathrm{~km}$ from Atar to Chinguetti (JMP); Ben Amira (JMP); between Ouarâne and Atar (Pleguezuelos et al., 2005); Chinguetti (Wilms \& Böhme, 2001); Choum, $40 \mathrm{~S}$ to Atar (JMP); Dahr Chinguetti (JMP); El Beyyed (JMP); Guelb er Richât (Wilms \& Böhme, 2001; JMP); Hamdoun (Dekeyser \& Villiers, 1956; Wilms \& Böhme, 2001); Iriji (Dekeyser \& Villiers, 1956); Molomhar (Dekeyser \& Villiers, 1956); Ouadâne (Wilms \& Böhme, 2001); Oued Choûm (JMP); Oued Séguelil (JMP); road Atar-Chinguetti via Ebnou pass (Brito, 2003); Te-n-Zak pass, road Choum-Atar (Brito, 2003); ca. $50 \mathrm{~km} \mathrm{~S}$ of Choum, road Choum-Atar (Brito, 2003); Dakhlet-Nouâdhibou: between Aghoueyyt and Inal (JMP); ca $5 \mathrm{~km}$ west of Inal, road from Nouadhibou-Choum (JMP); Tmeimichât (JMP); Hodh Ech Chargui: Oualâta (JMP); Inchiri: Akjoujt (JMP); Boudarga (JMP); Groutte Chauves-Souris, Guelb Moghrein (Jullien \& Petter, 1969 [1970]); Tagant: Guelta Matmata (Wilms \& Böhme, 2001); Lekhcheb (JMP); Tiris Zemmour: Bir Moghrein (Wilms \& Böhme, 2001); between Zouérat and Bir Moghrein (JMP; Pleguezuelos et al., 2005); between Zouerat and Rich (Pleguezuelos et al., 2005); Dayet el Aam (Donaire et al., 2000); Esfira (Pleguezuelos et al., 2005); Road N50 (Algeria), on the Mauritanian border (Donaire et al., 2000); Zemmour (Wilms \& Böhme, 2001); Zouérat (JMP).

Comments: Willms \& Böhme (2001) elevated Uromastyx acanthinura dispar to full species and Uromastyx acanthinura flavifasciata and U. mailensis were considered subspecies of the former. Assignation of specimens to species or subspecies of the Uromastyx acanthinura group have been commonly based on coloration and pholidosis (Mateo et al., 1998; Wilms \& Böhme, 2001; Brito, 2003; Geniez et al., 2004). But some of the described subspecies occur in simpatry, which is not compatible with the definition of subspecies (Mayr, 1942). Hence, either some cryptic species occur in sympatry or it is a case of intraspecific polymorphism. 


\section{CHAMALEONIDAE}

Chamaeleo africanus Laurenti, 1768

Distribution: Trarza: Diwaling National Park (Nickel, 2003).

Chamaeleo senegalensis Daudin, 1802

DisTRIBUTION: Mauritania, without locality (Grandison, 1956).

\section{GEKKONIDAE}

Hemidactylus brooki Gray, 1825

DisTRIBUTION: Dakhlet-Nouâdhibou: Nouâdhibou (JMP); Hodh Ech Chargui: Mahmûdé Lake (Nickel, 2003, JMP); Hodh El Gharbi: Zoueina (JMP); Nouakchott: Nouakchott (Ineich, 1997); Trarza: Chott Boul (Ineich, 1997); Dar es Salam (Ineich, 1997).

COMMENTs: One specimen more similar to Hemidactylus turcicus (Linnaeus, 1758) was found by JMP in a house at Nouâdhibou (Cap Blanc). This species has colonized distant areas (Bons \& Geniez, 1996) and this could well be the case in the Port of Nouâdhibou.

\section{Pristurus adrarensis Geniez \& Arnold, 2006}

DisTRIBUTION: Adrar: Zerga mountains, about $34 \mathrm{~km}$ WSW of Chinguetti (Geniez \& Arnold, 2006); 44 km from Chinguetti on the road to Adrar, $19 \mathrm{~km}$ before the Nouatil Pass (Geniez \& Arnold, 2006).

COMMENTS: endemic for the Adrar, Mauritania.

\section{Stenodactylus petrii Anderson, 1896}

Distribution: Adrar: Atar (Geniez et al., 2004); Ben Amira (JMP); Oued Choûm (JMP); DakhletNouâdhibou: Cap Blanc (Pleguezuelos et al., 2005); Oued Ech Chibka (Ineich, 1997); Tmeïmïchât (JMP); Inchiri: Agneïtîr (Ineich, 1997); Akchâr (Angel, 1938; Padial et al., 2002); Azeffâl (Ineich, 1997); Bennichchâb (JMP); dunes near Bennichchâb (Pleguezuelos et al., 2005); Nouakchott: Nouakchott (Ineich, 1997); Trarza: Tamzakt (Ineich, 1997); Tiris Zemmour: between Zouérat and Bir Moghrein (JMP); Beziah, Zemmour (Angel, 1938).

Comments: Böhme et al., (2001) noted that specimens of this species in SW Mauritania are more robust and intensively patterned dorsally than other populations.

Stenodactylus sthenodactylus (Lichtenstein, 1823)

DistriBution: Dakhlet-Nouâdhibou: Banc d'Arguin National Park (JMP); Cap Blanc (Böttger, 1921;
Geniez et al., 2004); Nouâdhibou (JMP); Oued Ech Chibka (Ineich, 1997); Inâl (Geniez et al., 2004); Hodh Ech Chargui: between Nema and Oualâta (JMP); Inchiri: Akchâr (Padial et al., 2002); Agneïtîr (Ineich, 1997); Azeffâl, Tânît (Ineich, 1997); Nouakchott: Nouakchott (Ineich, 1997); Trarza: Chott Boul (Ineich, 1997); Dar es Salam (Ineich, 1997). Tiris Zemmour: Beziah, Zemmour (Angel, 1938); Fdérik (Geniez et al., 2004).

Comments: Pellegrin (1910) cited this species as $S$. $s$. mauritanicus Guichenot, 1850 for two localities between Cap Blanc (Mauritania) and Sant Louis (Senegal): Boguent and Touail. Welch's (1982) record of this species for Mauritania is based on Pellegrin (1910).

Tarentola annularis (Geoffroy Saint-Hilaire, 1809)

Distribution: Adrar: Atar (Joger, 1980; Joger, 1984); Atar, 65 km S (JMP); Ben Amira (JMP); Chinguetti (Joger, 1984; Pleguezuelos et al., 2005); Choûm (Geniez et al., 2004); Ouadâne (Joger, 1984); Oued Hassi el Atil (Joger, 1980); Tintan (Pellegrin, 1910); $30 \mathrm{~km}$ E of Atar, road Atar-Chinguetti via Amogjar pass (Geniez \& Arnold, 2006); Dakhlet-Nouâdhibou: Banc d'Arguin National Park (Padial et al., 2002); Cap Blanc (Geniez et al., 2004); Inâl area (Geniez et al., 2004); Marzouba (Padial et al., 2002); Oued Ech Chibka (Ineich, 1997); Inchiri: Akchâr (Padial et al., 2002); Akjoujt (Joger, 1980; Joger, 1984); Bennichchâb (JMP); Châmi (JMP); Tijirît (JMP); Nouakchott: Nouackchott (Joger, 1984); Trarza: Tamzakt (Ineich, 1997); Tiris Zemmour: Bir Mogreïn (Joger, 1984); Fdérik (Fort Gouraud) (Joger, 1984).

CommENTs: Grandison (1961) included distributional information for Mauritania without names of localities.

\section{Tarentola chazaliae (Mocquard, 1895)}

DistriBUTION: Dakhlet-Nouâdhibou: Nouâdhibou (Mocquard, 1895; Pleguezuelos et al., 2005); Cap Blanc (Ineich et al., 1997, 1998; Geniez et al., 2004; Pleguezuelos et al., 2005); Boû Lanouar area (Geniez et al., 2004).

CommEnTs: Although this species was thought to range to Senegal throughout all the Mauritanian Coast (Schleich et al. 1996; Bons \& Geniez, 1996; Geniez et al. 2004), no confirmed record exists further south of Cap Blanc (Ineich et al., 1998).

Tarentola ephippiata O'Shaughnessy, 1875

Distribution: Adrar: Atar (Joger, 1984); between Tintane and Akjoujt (Pellegrin, 1910); L'Nouk Pass (Pleguezuelos et al., 2005); W from Ouadâne 
(Geniez et al., 2004) Assaba: Bougari (Nickel, 2003; JMP); Dakhlet-Nouâdhibou: Cap Blanc (Geniez et al., 2004); Marzouba (Padial et al., 2002); Nouâdhibou (JMP); Oued Ech Chibka (Ineich, 1997); Hodh El Gharbi: Zoueina (JMP); Ayoûn el Atroûs (Joger, 1984); Hodh Ech Chargui: Oualata (Joger, 1984); Inchiri: Akjoujt (Pellegrin, 1910; Joger, 1984); between Akjoujt and the Ocean (Angel, 1938); Châmi (JMP); Nouakchott: Nouakchott (Ineich, 1997); Tagant: Tidjikja (Joger, 1984); Tiris Zemmour: Bir Mogreïn (Angel, 1938); Zemmour, Beziah (Angel, 1938).

Comments: In Mauritania the subspecies T. e. hoggarensis (Joger, 1984) occurs. Grandison (1961) included some distributional information for Mauritania without locality names.

\section{Tarentola parvicarinata Joger, 1980}

Distribution: Adrar: Atar (Joger, 1980); Guelta Hamdoun (Joger, 1980); Oued Choûm (JMP); Oued Hassi el Atil (Joger, 1980); Terjît (JMP; Pleguezuelos et al., 2005); Tintane (Joger, 1980); Zerga mountains, about $34 \mathrm{~km}$ WSW of Chinguetti (Geniez \& Arnold, 2006); $30 \mathrm{~km} \mathrm{E} \mathrm{of} \mathrm{Atar,} \mathrm{road} \mathrm{Atar-Chinguetti} \mathrm{via}$ Amogjar pass (Geniez \& Arnold, 2006); Assaba: Bougari (Nickel, 2003); Guelta Oumm Lebare (JMP); Metraucha (Nickel, 2003); Guidimaka: Bouli (JMP); Hodh Ech Chargui: Oualâta (Joger, 1980); Hodh El Gharbi: Ayoûn El Atroûs (Joger, 1980); Tintâne (JMP); Inchiri: Akjoujt (Joger, 1980); Tagant: Lekhcheb (JMP); Tîchît (JMP); Tidjikja (JMP).

Tropiocolotes algericus (Loveridge, 1940)

DistriBUTION: Dakhlet-Nouâdhibou: Cap Blanc (Geniez et al., 2004); Inchiri: Agneïtîr (Ineich, 1997); Azeffâl (Ineich, 1997); Tiris Zemmour: Road N50 (Algeria), on the Mauritanian border (Donaire et al., 2000); Trarza: Tamzakt (Ineich, 1997).

COMMENTS: This species was previously considered a subspecies of $T$. tripolitanus.

\section{Tropiocolotes tripolitanus Peters, 1880}

Distribution: Adrar: Chinguetti (Dekeyser \& Villiers, 1956); Choum (Geniez et al., 2004); Ouadâne (JMP); Zerga mountains, about $34 \mathrm{~km}$ WSW of Chinguetti (Geniez \& Arnold, 2006); 30 km E of Atar, road AtarChinguetti via Amogjar pass (Geniez \& Arnold, 2006); Assaba: Bougari (Nickel, 2003); Hodh Ech Chargui: Oualâta (JMP); Hodh Ech Chargui: Mahmûdé Lake (Nickel, 2003); Hodh El Gharbi: Zoueina (JMP).

\section{LACERTIDAE}

Acanthodactylus aureus Günther, 1903

Distribution: Dakhlet-Nouâdhibou: Atar, near Nouâdhibou (Crochet et al., 2003); Birguendouz (JMP); Cap Blanc (Mocquard, 1895; Pellegrin, 1910; Böttger, 1921; Ineich, 1997; Crochet et al., 2003); Nouâdhibou (Salvador, 1982; Bons \& Geniez, 1996; Padial et al., 2002; Brito, 2003; Crochet et al., 2003); Inchiri: Akchâr (Padial et al., 2002); Akjoujt (Pellegrin, 1910); between Akjoujt and the Ocean (Salvador, 1982).

\section{Acanthodactylus boskianus (Daudin, 1802)}

Distribution: Adrar: Choum (Geniez et al., 2004); Kanoal, Oued Séguelil (Dekeyser \& Villiers, 1956); S from Atar (Geniez et al., 2004); Dakhlet-Nouâdhibou: between Nouâdhibou and Boû Lanouâr (JMP); Cap Timirist (Ineich, 1997); Inchiri: between Akjoujt and the Ocean (Angel, 1938; Salvador, 1982); Tiris Zemmour: Bir Moghrein; around $100 \mathrm{~km} \mathrm{~S}$ from Bir Moghrein (Geniez et al., 2004); Trarza: Muri (JMP); Nouakchott (Salvador, 1982; Ineich, 1997); Tamzakt (Ineich, 1997).

Acanthodactylus busacki Salvador, 1982

Distribution: Tiris Zemmour: Road N50 (Algeria) on the Mauritanian border (Donaire et al., 2000).

Acanthodactylus dumerili (Milne Edwards, 1829)

Distribution: Adrar: Atar (Salvador, 1982); Atar, 65 km S (JMP); Ain Savra (JMP); Tzougui (Salvador, 1982); Ben Amira (JMP); Chinguetti (Pleguezuelos et al., 2005); Ez Zerga (JMP); Oued Choûm (JMP); Tasiast, Ogla de Tamarat (Salvador, 1982); Zerga mountains, about $34 \mathrm{~km}$ WSW of Chinguetti (Geniez \& Arnold, 2006); $65 \mathrm{Km} \mathrm{SE}$ of Atar (JMP); $30 \mathrm{~km} \mathrm{E}$ of Atar, road Atar-Chinguetti via Amogjar Pass (Brito, 2003); Dakhlet-Nouâdhibou: Banc d'Arguin National Park (Padial et al., 2002); Mamghar (Angel, 1938; Salvador, 1982); Tafarit Cape (Crochet et al., 2003); Tidra Island (Crochet et al., 2003); Serreni, Banc D'Arguin National Park (Crochet et al., 2003); Guidimaka: Lake Houi (Salvador, 1982); Hodh Ech Chargui: Oualâta (JMP); Hodh El Gharbi: Ayoûn El Atroûss (JMP); Inchiri: Akjoujt (Salvador, 1982); Akjoujt, $20 \mathrm{~km} \mathrm{NW} \mathrm{(JMP);} \mathrm{Agneïtîr} \mathrm{(Crochet} \mathrm{et} \mathrm{al.,}$ 2003); Akchâr (Padial et al., 2002; Crochet et al., 2003); Tânît (Crochet et al., 2003); Aouarouâr, 30 km from Jreïf, Agneïtir (Crochet et al., 2003); Azeffâl (Crochet et al., 2003); Bennichchâb (JMP); between Akjoujt and the Ocean (Angel, 1938; Salvador, 1982); Manate, Agneïtir (Crochet et al., 2003); Tembrahim 
(Padial et al., 2002); Tijirît (Padial et al., 2002); Nouakchott: Nouakchott (Salvador, 1982); Trarza: Blaouakh (Crochet et al., 2003); Chott Boul (Crochet et al., 2003); Dar Es Salam (Crochet et al., 2003); El Hazra (Crochet et al., 2003); Hassi Loubbou, El Mhaïjrât, (Crochet et al., 2003); Lekeseyr, $7.5 \mathrm{~km} \mathrm{~N}$ of (Crochet et al., 2003); Muri (JMP); Nouakchott, 10 $\mathrm{km} \mathrm{N}$ of (Crochet et al., 2003); Nouakchott, $12 \mathrm{~km} \mathrm{~N}$ of (Crochet et al., 2003); Tamzakt (Crochet et al., 2003); Tanit (Crochet et al., 2003); Tiouilit (Crochet et al., 2003; JMP); Tagant: Lekhcheb (JMP); Lekhcheb, $25 \mathrm{~km} \mathrm{~S}$ (JMP); road between Atar and Tidjikja (JMP); Tidjikja (Salvador, 1982); Zig, between Lekhcheb and Tîchît (JMP); Tiris Zemmour: between Zouérat and Bir Moghrein (JMP); Zouérat, 60 km S (JMP); Bir Moghrein; Fdérik (Salvador, 1982); Toûâjil (JMP).

COMMENTS: Boulenger (1921) already mentioned this species for Mauritania. Old records of this species could contain different currently recognized species. Crochet et al., (2003) cited cases of hybridization between $A$. dumerili and $A$. senegalensis. Records of Ineich (1997) were subsequently studied and specifically relocated by Crochet et al., (2003); therefore I only refer to the later reference.

\section{Acanthodactylus longipes Boulenger, 1921}

Distribution: Adrar: Amguili, Assabet and Meddahiya (Salvador, 1982); Choûm (Salvador, 1982); Chinquetti (Salvador, 1982); Ijâfen area (BM 1975.1398; Salvador, 1982); Ogueïlet Meiag (Salvador, 1982); Oued Choûm (JMP); 50 km S of Choum, road ChoumAtar (Brito, 2003); Tacarât area (BM 1975-1401; Salvador, 1982); Dakhlet Nouâdhibou: Iouik (Crochet et al., 2003); St-Jean Island, Banc D'Arguin NP (Crochet et al., 2003); Inchiri: Agneïtir (Crochet et al., 2003); Azeffâl (Crochet et al., 2003); Tagant: Adâfer Meridional (Salvador, 1982); Trarza: Akreïdil, $50 \mathrm{~km}$ NE Nouakchott (Crochet et al., 2003); Chott Boul, 6 $\mathrm{km} \mathrm{S}$ of (Crochet et al., 2003); El Hazra (Crochet et al., 2003).

Acanthodactylus scutellatus (Audouin, 1827)

Distribution: Adrar: E of Ouadane (Geniez \& Luch, 2004); Tiris Zemmour: Toûâjil (JMP).

COMMENTS: The first country record was reported by Geniez \& Luch (2004) and assigned to A. scutellatus audouini. Specimens from Toûâjil are deposited in MNCN.

Acanthodactylus senegalensis Chabanaud, 1918

Distribution: Dakhlet Nouadhibou: Tafarit Cape (Crochet et al., 2003); Inchiri: Akchâr (Crochet et al.,
2003); Trarza: Chott Boul (Crochet et al., 2003); Dar Es Salam (Crochet et al., 2003); Hasseï Gáboûn (Crochet et al., 2003); Muri (JMP); Nouakchott (Crochet et al., 2003); $10 \mathrm{Km} \mathrm{N}$ of Nouakchott (Crochet et al., 2003); $12 \mathrm{~km} \mathrm{~N}$ of Nouakchott (Crochet et al., 2003); $156 \mathrm{~km}$ beyond Nouakchott towards Rosso (Crochet et al., 2003); Tamzakt (Crochet et al., 2003); 4 Km NW Tamzakt (Crochet et al., 2003); Tanit (Crochet et al., 2003).

COMMENTS: Some previous records of $A$. aureus from coastal Mauritania may correspond to $A$. senegalensis. Records of Ineich (1997) were subsequently studied and specifically relocated by Crochet et al., (2003), I therefore refer to the later.

Acanthodactylus taghitensis Geniez \& Foucart, 1995

Distribution: Tiris Zemmour: Fdérik (Hasi et al., 1997; Crochet et al., 2003); Northern Tagenzé, Ouan n'Namour (Geniez \& Luch, 2004).

Latastia longicauda (Reuss, 1834)

Distribution: Trarza: Chott Boul (Ineich, 1997); Dar es Salam (Ineich, 1997).

Mesalina guttulata (Lichtenstein, 1823)

Distribution: Adrar: road Atar-Chinguetti via Ebnou Pass (Brito, 2003); Tiris Zemmour: Fdérik (Geniez et al., 2004).

Comments: Grandison (1956) did not report any locality for this species and, therefore, the record of Brito (2003) and Geniez et al. (2004) represent the first precise records of this species for the country.

Mesalina olivieri (Adouin, 1829)

Distribution: Adrar: Chinguetti (Dekeyser \& Villiers, 1956); southern limit of Adrar area (Geniez et al., 2004); Inchiri: Akjoujt (Böhme et al., 2001); Tagant: Lekhcheb (JMP).

COMmENTs: Grandison, 1956 cited this species without locality.

\section{Mesalina pasteuri (Bons, 1960)}

Distribution: Adrar: Oued Choûm (JMP); Inchiri: Tembrahim, Akchâr (Padial et al., 2002); Tagant: Lekhcheb (JMP); Tiris Zemmour: Aghreijît area (Geniez et al., 2004); Châr area (Geniez et al., 2004).

Mesalina rubropunctata (Lichtenstein, 1823)

DistriBution: Adrar: El Beyyed (JMP); Choûm (Böhme et al., 2001); Inchiri: Akjoujt (Böhme et al., 2001); Tiris Zemmour: Dayet el Am (Donaire et al., 2000). 


\section{SCINCIDAE}

Chalcides ocellatus (Forskal, 1775)

DisTRIBUTION: Adrar: Chinguetti (Dekeyser \& Villiers, 1956); Hodh El Gharbi: Ayoûn El Atroûs (JMP).

\section{Scincopus fasciatus Peters, 1864}

DisTRIBUTION: Adrar: Nterguent, near Atar (Geniez et al., 2004); Trarza: near Tiguent (Böhme et al., 2001).

COMMENTs: Welch (1982) already cited this species without locality.

\section{Scincus albifasciatus Boulenger, 1890}

Distribution: Adrar: Atar (Arnold \& Levinton, 1977); Ain Savra (JMP); Ben Amira (JMP); Chinguetti (Arnold \& Levinton, 1977); Choûm (Arnold \& Levinton, 1977); Legleitât (Arnold \& Levinton, 1977); Kanoal, Oued Séguelil (Dekeyser \& Villiers, 1956); Oued Aïmou (Angel, 1938); Oued Choûm (JMP); Zerga mountains, about $34 \mathrm{~km} \mathrm{WSW}$ of Chinguetti (Geniez \& Arnold, 2006); Dakhlet-Nouâdhibou: Boû Lanouâr (JMP); Inâl (Geniez et al., 2004); Tassiast (Arnold \& Levinton, 1977); Hodh El Gharbi: Ayoûn El Atroûs (JMP); Inchiri: Akchâr, Tânît (Ineich, 1997); Agneïtîr (Ineich, 1997); Azeffâl (Ineich, 1997); Boudarga (JMP); Nouakchott: Nouakchott (Arnold \& Levinton, 1977); Tiris Zemmour: N from Fdérik (Geniez et al., 2004); Tourîne area (Geniez et al., 2004); Trarza: without locality (Arnold \& Levinton, 1977).

Comments: Arnold and Levinton (1977) considered most Mauritanian populations as S. scincus albifasciatus, but they found differences in colour pattern among specimens from the Adrar region, even in the same locality.

\section{Sphenops delislii (Lataste, 1876)}

Distribution: Adrar: Chinguetti (Dekeyser \& Villiers, 1956); Dakhlet Nouâdhibou: road from Boû Lanouâr to Choum, around Inâl and Tmeïmîchât (Geniez et al., 2004).

\section{Sphenops sphenopsiformis (Duméril, 1856)}

Distribution: Dakhlet-Nouâdhibou: Cap Blanc (Grandison, 1956; Salvador, 1982; Welch, 1982; Le Berre, 1989; Bons \& Geniez, 1996; Ineich, 1997; Geniez et al., 2004); Inchiri: Akjoujt (Pellegrin, 1910).

Trachylepis perrotetii (Duméril \& Bibron, 1839)

DisTRIBUTION: Trarza: Chott Boul (Ineich, 1997); Dar es Salam (Ineich, 1997).

\section{VARANIDAE}

Varanus exanthematicus (Bosc, 1792)

Distribution: Hodh El Gharbi: Foulania (JMP); Trarza: Dar es Salam (Ineich, 1997).

Comments: Böhme (2003) also mentions V. exanthematicus for Subsaharan Mauritania.

Varanus griseus (Daudin, 1803)

Distribution: Adrar: Kanoal, Oued Séguelil (Dekeyser \& Villiers, 1956); Ouadane area (Geniez et al., 2004); Oued Choûm (JMP); Tin Demane (Geniez et al., 2004); 20 ${ }^{\circ} 15^{\prime} \mathrm{S} / 13^{\circ} 16^{\prime}$ (Pleguezuelos et al., 2005); Dakhlet-Nouâdhibou: Cap Blanc (Böttger, 1921; Geniez et al., 2004); Hodh El Gharbi: Ayoûn El Atroûs (JMP); Inchiri: Bennichchâb (JMP); Chekraf (Padial et al., 2002); S from Akjoujt (Böhme et al., 2001); Nouakchott: N from Nouakchott (Ineich, 1997); Tagant: Lekhcheb (JMP); Trarza: Boutlimit (JMP); Tamzakt (Ineich, 1997).

Comments: $V . g$. griseus is the subspecies present in Mauritania (Böhme, 2003).

Varanus niloticus Linnaeus, 1766

Distribution: Assaba: Bou Bleï'îne (JMP); Bougari (Nickel, 2003); Hodh Ech Chargui: Mahmûdé Lake (Nickel, 2003); Hodh El Gharbi: Zoueina (JMP); Tagant: Guelta Fanar (JMP); Guelta Matmata (JMP); Trarza: Chott Boul (Ineich, 1997); Dar es Salam (Ineich, 1997); road Diama-Rosso (Brito, 2003).

Comments: This species can be found in isolated wetlands surrounded by dunes in the Assaba region.

\section{SERPENTES}

\section{LEPTOTYPHLOPIDAE}

Leptotyphlops algeriensis (Jaquet, 1896)

Distribution: Adrar: Kanoal, Oued Séguelil (Dekeyser \& Villiers, 1956).

Comments: this species was considered a synonym of $L$. macrorhynchus until very recently (Hahn \& Wallach, 1998), but it was subsequently resurrected by Trape (2002).

Leptotyphlops boueti (Chabanaud, 1917)

Distribution: Brakna: Aleg (Villiers, 1950); Hodh El Gharbi: Aioûn el Atroûs (Villiers, 1950).

COMMENTS: cited by Villiers as L. narirostris boueti following the opinion of A. Loveridge [see Remarks on Hahn \& Wallach (1998) for this species]. 
Nevertheless, the later authors mentioned significant differences between specimens of $L$. narirostris and $L$. boueti. Trape (2002) used the combination L. boueti. Under this author's view, the species shows differential characters [following Hahn \& Wallach's, (1998) observations] that support its specific status.

Leptotyphlops cairi (Dumeril \& Bribon, 1844)

Distribution: Trarza: without locality (BMNH 1913.5.9.45) (Hahn \& Wallach, 1998).

CommenTs: Welch (1982) listed this species without locality for the country, probably on the basis of the single specimen collected by Audan and deposited in the British Museum of Natural History.

\section{TYPHLOPIDAE}

Typhlops etheridgei Wallach, 2002

DisTRIBUTION: Adrar: between Atar and Choum (Wallach, 2002).

COMmENTs: this species is endemic for Mauritania and only known from the type locality (between Atar and Choûm along Route Nationale 1, a distance of ca. 85 air $\mathrm{km}$ through the Amsâga region, western Adrar Region, west-central Mauritania).

\section{BOIDAE}

Gongylophis muelleri Boulenger, 1892

DISTRIBUTION: Inchiri: between Akjoujt and the ocean (Angel, 1938); Tasiast, Oued Aïmou (Angel, 1938); Trarza: Bafrechié (Villiers, 1954); R'kiz (Villiers, 1954); without locality (Boulenger, 1919).

COMMENTS: Angel (1938) described E. m. subniger from Tasiast, Oued Aïmou. Villiers (1954) detected some morphological differences between the specimens from Mauritania studied by him and this subspecies. Ineich (1997) cited this species for the Mauritanian coast without locality.

\section{ATRACTASPIDIDAE}

\section{Atractaspis microlepidota Günther, 1866}

Distribution: Trarza: without locality (Boulenger, 1919, Angel, 1933, Villiers, 1950, 1951)

COMMENTS: Many authors have cited this species for Mauritania (Boulenger, 1919; Angel, 1933; Villiers, 1950; Villiers, 1951; Villiers, 1975; Welch, 1982; Hughes, 1983; Le Berre, 1989; Chippaux, 2001), but none have given any precise locality.
Atractaspis micropholis Gunther, 1872

Distribution: Hodh Ech Chargui: Adel Bagrou (H. Nickel pers. comm.)

Comments: Welch (1982) cited this species without locality.

\section{COLUBRIDAE}

Hemorrhois algirus (Jan, 1863)

DisTRIBUTION: Dakhlet-Nouâdhibou: Nouâdhibou (Chabanaud, 1924; Monod, 1928); Tiris Zemmour: between Zemmour and Bir Moghrein (Geniez et al., 2004); Fdérik (Villiers, 1950); aprox $100 \mathrm{~km} \mathrm{~S}$ from Bir Moghrein (Geniez et al., 2004).

Comments: $H$. a. intermedius is the subspecies present in Mauritania.

Hemorrhois dorri (Lataste, 1888)

Distribution: Assaba: Metraucha (Nickel, 2003).

Dasypeltis scabra (Linnaeus, 1759)

DistRIBUTION: Trarza: Tamzakt (Ineich, 1997); Dar es Salam (Ineich, 1997).

Lamprophis fuliginosus (Boié, 1827)

Distribution: Nouakchott: Nouakchott (Thorpe \& McCarthy, 1978); Trarza: without locality (Boulenger, 1919; Villiers, 1950).

Lytorhynchus diadema (Duméril, Bibron \& Duméril, 1854)

Distribution: Adrar: Kanoal, Oued Séguelil (Dekeyser \& Villiers, 1956); Dakhlet-Nouâdhibou: Nouâdhibou (Villiers, 1950); Inâl (Geniez et al., 2004); Isnaía, near Cap Timirist (Villiers, 1954); Inchiri: Akjoujt (Pellegrin, 1910, 1911); Azeffâl (Geniez et al., 2004); Nouakchott: Nouakchott (Ineich, 1997); Tagant: Bir el Fouj, NW Tîchît (Villiers, 1956); Tiris Zemmour: W from Touâjîl, Aghreijît area (Geniez et al., 2004); Trarza: Tamzakt (Ineich, 1997).

Prosymna meleagris (Reinhardt, 1843)

DisTRIBUTION: Mauritania, without locality (Welch, 1982).

Scutophis moilensis (Reuss, 1834)

DistriBution: Adrar: El Berbera (Villiers, 1950, 1951); Dakhlet-Nouâdhibou: El Aïouej, NW Mauritanien, 60 Km NE from Port Etienne/Nouadhibou (Angel, 1939); Inchiri: between Akjoujt and the Ocean (Angel, 1938); Nouakchott: Nouakchott; Tiris Zemmour: between 
Fdérik and Bir Moghrein (Geniez et al., 2004); Bir Moghrein (Geniez et al., 2004); Châr area (Geniez et al., 2004); Fdérik (Villiers, 1950b); Trarza: Tamzakt (Ineich, 1997).

CoMments: Boulenger (1919) cited Malpolon mospessulanus for "borders of the Mediterranean, southwards to Mauritania". Villiers (1950) considered this record doubtful, and suggested that Boulenger's specimen may have been found in South Morocco. This species reaches Dakhla (West Sahara).

\section{Psammophis elegans Shaw, 1802}

Distribution: Assaba: Metraucha (Nickel, 2003); Trarza: cited by Boulenger (1919), without locality (see also Villiers, 1950, 1951).

COMMENTS: Although many authors cited this species for Mauritania, the only precise record corresponds to that of Nickel (2003) for the Assaba region. The taxonomic status and geographical assignation of records to species of the genus Psammophis is problematic, see (Chippaux, 2001) and Trape and Mané (2002).

Psammophis phillipsi (Hallowell, 1844)

Distribution: Trarza: Chott Boul (Ineich, 1997).

Comments: See comments on $P$. elegans.

\section{Psammophis schokari (Forskal, 1775)}

Distribution: Adrar: Atar (Villiers, 1950); Oued Choûm (JMP); Dakhlet-Nouâdhibou: Nouâdhibou (Chabanaud, 1924; Monod, 1928; Bons \& Geniez, 1996); Inchiri: Agneïtîr (Ineich, 1997); Azeffâl (Padial et al., 2002); Akjoujt (Angel, 1938; Loveridge, 1941); between Akjoujt and the Ocean (Angel, 1938); Nouakchott: Nouakchott (Ineich, 1997); Tiris Zemmour: Bir Mogrein (Geniez et al., 2004); Châr area (Geniez et al., 2004); Trarza: Chott Boul (Ineich, 1997); near the Senegal border (Ineich, 1997); Tamzakt (Ineich, 1997); without locality (Boulenger, 1919; Villiers, 1950).

Comments: Loveridge (1941) cited P. schokari as $P$. sibilans schokari from Akjoujt. Angel \& Lothe (1938) cited this species for Mauritania without locality. See comments on $P$. elegans.

\section{Psammophis sibilans (Linnaeus, 1758)}

Distribution: Trarza: Villiers (1950) mentioned two specimens from Trarza deposited in the BM. See comments on P. elegans.

Spalerosophis diadema (Schlegel, 1837)

Distribution: Adrar: Atar (Villiers, 1950; Geniez et al., 2004); Trarza: Tamzakt (Ineich, 1997).
Telescopus guidimakaensis (Chabanaud, 1916)

DisTRIBUTION: Guidimaka: without locality (typus of Tarbophis guidimakaensis) (Chabanaud, 1916; see also Villiers, 1950); Trarza: without locality (Boulenger, 1919).

Comments: Cited as T. obtusus (Reuss, 1834) for Trarza region without locality by Boulenger (1919) and subsequent authors (Villiers, 1950; Villiers, 1975; Welch, 1982; Le Berre, 1989; Hasi et al., 1997; Chippaux, 2001), probably followed Boulenger's record. Welch (1982) cited this species as Telescopus dhara obtusus. Geniez et al. (2004) cited this species for Ichargan, in the Zemmour Mountains near the Mauritanian border. Hence, this species is expected to occur also in northern Mauritania.

Ramphiophis oxyrynchus (Rheinhardt, 1843)

Distribution: Trarza: Diwaling National Park (Nickel, 2003).

\section{ELAPIDAE}

Elapsoidea semiannulata Bocage, 1882

DisTRIBUTION: Guidimaka: without locality (Angel, 1933).

COMMENTS: cited by Angel (1933) as E. sundevalli guntherii (see also Villiers, 1950).

Naja katiensis Angel, 1922

Distribution: "Sud de la Mauritanie" (Villiers, 1975).

Naja nigricollis Reinhardt, 1843

Distribution: Gorgol: M'bout (Villiers, 1950); Nouakchott: Nouakchott (Villiers, 1950); Trarza: Boutlimit (Villiers, 1950).

Comments: Villiers (1951) gives the same records.

\section{PYTHONIDAE}

Python sebae Gmelin, 1788

Distribution: Assaba: Bougari (Nickel, 2003); Metraucha (Nickel, 2003); Gorgol: M'bout (Villiers, 1950); Guidimaka: Boudami (Villiers, 1950); Meyane (Villiers, 1950); Hodh Ech Chargui: Mahmûdé Lake (Nickel, 2003; Padial, 2003); Tagant: Tamourt en Naaj (Padial, 2003); Trarza: $20 \mathrm{~km} \mathrm{~N}$ of N'Diago (Padial, 2003), Diawling National Park (Ineich, 1997); R'Kiz (Villiers, 1950).

Comments: see Padial (2003) for a recent revision on the status of this species in Mauritania. 


\section{VIPERIDAE}

Causus maculatus (Hallowell, 1842)

DistriBution: Trarza: without locality (Chabanaud, 1916).

Comments: Cited by Chabanaud (1916) and subsequently by Angel and Lothe (1938) and Villiers (1950) as Causus rhombeatus.

\section{Cerastes cerastes (Linnaeus, 1758)}

DisTRIBUTION: Adrar: Aouelloul (Villiers, 1950, 1951); Atar (Villiers, 1951); between Tmeïmîchât and Choûm (Geniez et al., 2004); Choûm (Geniez et al., 2004); Kanoal, Oued Séguelil (Dekeyser \& Villiers, 1956); Oued Choûm (JMP); Dakhlet-Nouâdhibou: between Boû Lanouâr and Inâl (Geniez et al., 2004); Marzouba (Padial et al., 2002); Nouâdhibou (Böttger, 1921); Tanoudert (Angel, 1939); Gorgol: M'Bout (Villiers, 1950); Inchiri: Akchâr, Tânît (Ineich, 1997); Akjoujt (Angel, 1938, Villiers, 1950); Aleibataf (Pellegrin, 1910); between Akjoujt and the ocean (Angel, 1938); Nouakchott: Nouakchott (Ineich, 1997); Tagant: Zig, between Lekhcheb and Tîchît (JMP); Tiris Zemmour: Bir Mogrein (Geniez et al., 2004); Fdérik (Villiers, 1950); Hasi el Fogra (Donaire et al., 2000); Trarza: Aleib Ataf (Pellegrin, 1911); Boutlimit (Villiers, 1950); Tamzakt (Ineich, 1997).

\section{Cerastes vipera (Linnaeus, 1758)}

Distribution: Adrar: between Tmeïmîchât and Choûm (Geniez et al., 2004); Chinguetti (Villiers, 1950; Dekeyser \& Villiers, 1956); Oued Choûm (JMP); Ouadane area (Geniez et al., 2004); Southern Adrar (Geniez et al., 2004); Dakhlet-Nouâdhibou: Boû Lanouâr area (Bons \& Geniez, 1996); Inchiri: Agneïtîr (Ineich, 1997); Akchâr, Tânît (Ineich, 1997); Aleibataf (Pellegrin, 1910); Azeffâl (Ineich, 1997); Bennichchâb, $25 \mathrm{~km} \mathrm{~S}$ (JMP); between Akjoujt and the ocean (Angel, 1938); Nouakchott: Nouakchott (Ineich, 1997); Tiris Zemmour: El Hammâmi area (Geniez et al., 2004); Tagant: Lekhcheb (JMP).

Comments: Angel \& Lothe (1938) cited this species for Saharan Mauritania.

Bitis arietans (Merrem, 1820)

Distribution: Gorgol: M'Bout (Villiers, 1950); Guidimaka: Sélibabi (Villiers, 1950); Trarza: Boutlimit (Villiers, 1950).

Echis leucogaster Roman, 1972

Distribution: Adrar: Atar (Villiers, 1950); Oued El Akhdar (Villiers, 1950, 1951); El Ouedï; Guelta
Hamdoun (Dekeyser \& Villiers, 1956); Iriji (Dekeyser \& Villiers, 1956); Hodh Ech Chargui: without concrete locality (Chippaux, 2001); Hodh El Gharbi: Ayoûn El Atroûs (JMP); Oumm El Khez (JMP); Trarza: between Nouakchott et Saint-Louis (Villiers, 1954); Marais de Toumboss (Villiers, 1954), près Keur Massène (Villiers, 1954); R'kiz (Villiers, 1954).

COMMENTS: Another possible record is from northern Mauritania, in Kreyma el Maijat, between Houmat and Doloa (Aymerich et al., 2004; Geniez et al., 2004).

Echis ocellatus Stemmler, 1970

DisTRIBUTION: Trarza: without locality (Villiers, 1950; Chippaux, 2001).

Comments: Most of these records may correspond to $E$. leucogaster. Those from Trarza have been cited as $E$. ocellatus by Chippaux (2001). Villiers (1950) cited it as E. carinatus.

\section{CROCODYLIA CROCODYLIDAE}

Crocodylus cataphractus Cuvier, 1825

Distribution: Senegal River (Fuchs et al., 1974; Villiers, 1958).

Comments: Specimens cited for Guelta Matamata of Tagant (Staudinger, 1929) correspond to C. suchus.

Crocodylus suchus (Geoffroy Saint-Hilaire, 1807)

DistriBUtion: Assaba: Bougari (Nickel, 2003); Metraucha (Nickel, 2003); Hodh Ech Chargui: Lake Dendaré; Tagant: Amzouzef (Behra, 1994); Ederoum (Behra, 1994); Guelta Fanar (JMP obs), Guelta Matmata (Staudinger, 1929; Behra, 1994; JMP); Laout (Behra, 1994); Rh Zembou (Behra, 1994).

Comments: Crocodiles were cited historically for Mauritania. For example in Lake Calula and M'bout in the Wilaya Gorgol, Lake Dendaré in Hodh Ech Chargui, and in Hodh El Gharbi (Pellegrin, 1911; Monod, 1921; Staudinger, 1929; Spatz, 1930; Joleaud, 1933; Villiers, 1958; Le Berre, 1989). Most records corresponded to Tagant and Assaba, where around 28 Gueltas still harbour crocodiles (Shine et $a l ., 2001)$. We herein report those localities where the presence of this species was confirmed recently (see: Behra, 1994; Shine et al., 2001 and Nickel, 2003 for updated information). Phylogenetic analyses (Schmitz et al., 2003) indicate that West African populations currently considered $C$. niloticus correspond to C. suchus (Geoffroy, 1807) while C. niloticus is restricted to East Africa. 


\section{TESTUDINES}

\section{CHELONIIDAE}

\section{Caretta caretta (Linnaeus, 1758)}

Distribution: Dakhlet-Nouâdhibou: Baie du Cansado (Maigret, 1983; Arvy et al., 1996); Baie du Lévrier (Maigret, 1983; Arvy et al., 1996); Banc d'Arguin National Park; Cap Blanc (Bons \& Geniez, 1996); Nouâdhibou (Maigret, 1983; Arvy et al., 1996); Pointe des Coquillages (Maigret, 1983; Arvy et al., 1996); Pointe des Crabes (Maigret, 1983; Arvy et al., 1996).

COMMENTS: Bons \& Geniez (1996) also reported the information of Maigret (1983). Arvy \& Dia (1997) reported this species for most of all the coastal area of Mauritania.

Chelonia mydas (Linnaeus, 1758)

Distribution: Dakhlet-Nouâdhibou: Baie d'Arguin (Maigret, 1983; Arvy et al., 1996); Baie du l'Etoile (Maigret, 1983; Arvy et al., 1996); Baie du Lévrier (Maigret, 1983; Arvy et al., 1996); Banc d'Arguin National Park (Padial et al., 2002); Cap Arguin (Maigret, 1975); Ile Marguerite (Maigret, 1983; Arvy et al., 1996); Iouîk (Maigret, 1983; Arvy et al., 1996); Nouâdhibou (Maigret, 1983; Arvy et al., 1996); Inchiri: El Mhaïjrât (Arvy et al., 1996); Jraïf (Arvy et al., 1996); Trarza: Blaouakh (Arvy et al., 1996); Campament 28 (Arvy et al., 1996); Campament 65 (Arvy et al., 1996); Nouakchott (Arvy et al., 1996).

Comments: Geniez \& Bons (1996) cited this species for all the Cap Blanc area. Arvy \& Dia (1997) reported this species for all the Mauritanian coast.

\section{Eretmochelys imbricata (Linnaeus, 1766)}

Distribution: Dakhlet-Nouâdhibou: Iouîk (Maigret, 1983; Arvy et al., 1996); Nouâdhibou (Maigret, 1983; Arvy et al., 1996); Trarza: Blaouakh (Maigret, 1983; Arvy et al., 1996); Campament 65 (Maigret, 1983; Arvy et al., 1996); El Msid (Maigret, 1983; Arvy et al., 1996); Nouakchott: Port of Nouakchott (Arvy et al., 1996).

Comments: Arvy \& Dia (1997) also reported this species for the coastal area (Grand Plage).

Lepidochelys kempii (Garman, 1880)

DisTRIBUTION: Nouakchott: Port of Nouakchott (Arvy et al., 1996; Arvy \& Dia, 1997); Trarza: Campament 28 (Arvy et al., 1996).

Lepidochelys olivacea (Eschscholtz, 1829)

Distribution: Dakhlet-Nouâdhibou: Nouâdhibou (Villiers, 1958; Bons \& Geniez, 1996); Nouâmghâr (Arvy \& Dia, 1997).

\section{DERMOCHELYIDAE}

Dermochelys coriacea (Vandelli, 1761)

DistriBution: Dakhlet-Nouâdhibou: Baie de Cansado (Maigret, 1983; Arvy et al., 1996; JMP); Baie de Etoile (Maigret, 1983; Arvy et al., 1996); Baie du Repos (Maigret, 1983; Arvy et al., 1996); Bahie du Lévrier (Maigret, 1983; Arvy et al., 1996; Arvy \& Dia, 1997); Plage SIGP (Maigret, 1983; Arvy et al., 1996); Cap Timirist (Maigret, 1983; Arvy et al., 1996).

COMMENTS: JMP and R. García Romo released an adult specimen accidentally trapped in an abandoned net in Cansado Bay, near Nouadhibou. Bons \& Geniez reported the records of Maigret $(1975,1983)$ for Cap Blanc area.

\section{EMYDIDAE}

\section{Mauremys leprosa (Scheweiger, 1812)}

DisTRIBUTION: Adrar: without locality (Villiers, 1958; Le Berre, 1989; Geniez et al., 2004); Tiris Zemmour: Idjil, near Fdérik (Villiers, 1958; Le Berre, 1989; Geniez et al., 2004); near Western Sahara border (Loveridge \& Williams, 1957).

COMments: These localities are isolated wetlands in Saharan Mauritania. People of Fdérik mentioned that the only wetland of the region disappeared due to water pumping for iron extraction. This activity may have led to the extinction of this terrapin population. There is no recent record of this species for the country but its presence in the Adrar or adjacent areas is still hoped for. Nevertheless, Dekeyser \& Villiers (1956) did not record this species there.

\section{PELOMEDUSIDAE}

Pelusios adansoni (Schweigger, 1812)

Distribution: Senegal River (Iverson, 1992).

COMMENTS: no concrete record in known.

Pelomedusa subrufa (Bonnaterra, 1789)

DistriBution: Senegal River (Iverson, 1992)

COMMENTS: no concrete record is known.

\section{TESTUDINIDAE}

Geochelone sulcata (Miller, 1779)

Distribution: Assaba: Kiffa (Arvy et al., 1997); Brakna: Aleg (Arvy et al., 1997); Bogué (Arvy et al., 1997); 
Guidimaka: Sélibabi (Arvy et al., 1997); Nouakchott: Nouakchott (Joger, 1981; Arvy et al., 1997); Tagant: Massif montagneux du Sud du Tagant (Arvy et al., 1997); Trarza: Amnéguir (Arvy et al., 1997); Boutlimit (Arvy et al., 1997); Hasseida (Arvy et al., 1997); Méderdra (Arvy et al., 1997); Parc National du Diawling et Dune littorale (Arvy et al., 1997); Rosso (Arvy et al., 1997); Tiguent (Arvy et al., 1997).

\section{TRIONYCHIDAE}

Cyclanorbis senegalensis (Duméril \& Bibron, 1835)

Distribution: Senegal River (Iverson, 1992; Joger \& Lambert, 1996).

COMMENTS: no concrete record is known.

Trionyx triunguis (Forskal, 1775)

DistRibution: Senegal River (Iverson, 1992).

COMMENTS: no concrete record is known.

\section{Discussion}

Some of the species cited in the literature or in the Internet databases should be removed from the lists. For example, Psammodromus algirus (Linnaeus, 1766) ranges from southern France to South Morocco (Bons \& Geniez, 1996). The record for Mauritania without locality (see Welch, 1982) is surely an error and I therefore exclude this species from the list. Saurodactylus brosseti Bons \& Pasteur, 1957, cited for the Banc d'Arguin (Mahe, 1985 in Geniez \& Bons, 1996), was surely mistaken for Tropiocolotes tripolitanus (Geniez \& Bons, 1996). However, S. brosseti may occur in northern Mauritania since it has been cited for Zemmour and Tindouf (Geniez et al., 2004). Ptyodactylus hasselquistii hasselquistii (Donndorff, 1798) was cited for Mauritania without locality (Welch, 1982). In a revision of this group, Heimes (1987) did not cite this species for the country and the nearest record in his distribution map corresponds to $P$. $h$. ragazzi from central Mali. Although the presence of Ptyodactylus is likely, I prefer to exclude this genus from the list until there is additional evidence of its presence in the country. Chabanaud (1917) cited Tarentola delalandii for Saharan Mauritania, a species that is restricted to the Canary Islands. Although we cannot discard an accidental reintroduction of this species in coastal Mauritania, I prefer to exclude this species from the list until confirmation. The record for Typhlops punctatus (Leach, 1819) by Wallach (2002) from the dry savanna of southern Mauritania is a mistake (Wallach, pers. com.). This species has not been recorded for the country to date, although its presence is to be expected because it occurs in northern Senegal (Roux-Estève, 1974). Malpolon monspessulanus and Macroprotodon cucullatus (Geoffroy St. Hilaire, 1827) were cited by Boulenger (1919) based on collections from Trarza made by Audan (see Villiers, 1950), we consider these records as erroneous, as did Villiers (1950) when he tentatively assigned these records to southern Morocco. The presence of Naja haje (Linnaeus, 1758) is not confirmed although it is listed in the EMBL reptile database. Villiers (1950) considered the presence of this species very likely. It occurs in Senegal and Mali, and Chippaux (2001) also included Mauritania within the range of this species without giving any concrete record. Bons \& Geniez (1996) and Geniez et al. (2004) cited this species for two localities in West Sahara, very close to the Mauritanian border. The absence of any concrete record for Mauritania is most likely due to a lack of sampling. Pelusios castaneus (Schweigger, 1812) is cited in the EMBL reptile database, but we were not able to locate any concrete record or reference for this species in Mauritania and, therefore, I tentatively exclude this species from the list. Mabuya quinquetaeniata (Lichtenstein, 1823) is listed in the WCMC web site for Mauritania without a concrete reference for corroboration. Nevertheless, this species inhabits adjacent areas in Mali (Joger \& Lambert, 1996) and its presence in Mauritania is expected.

The reptile fauna of Mauritania is currently composed of 86 species belonging to 21 families (Table 1). Lacertidae and Colubridae are the most diverse families, and Agamidae, Gekkonidae, Scincidae or Viperidae are also well represented. Among the 80 continental species, 47.5\% are Saharan, 33.8\% Afrotropical, 16.2\% Sahelian and $2.5 \%$ Mediterranean. The marine turtles form another important group, with six species. These data contrast with the 53 species (of which only 48 are valid) reported in the most complete and useful Internet database for reptiles (EMBL Reptile Database, 2006). Another database (WCMC, 2006) offers a list of 73 species (of which 65 are valid). The herpetological diversity of Mauritania is in urgent need of a thorough revision, as evidenced by the panorama of reptile diversity reported here.

Among the species expected to occur, at least nine may be found in Saharan environments, fourteen in the Sahelian savannah and two could have been potentially reintroduced. The expected species are as follows: a) Saharan species: Uromastyx acanthinura 
Table 1.- Number and percentage of species for each family of Mauritanian reptiles.

Tabla 1.- Número y porcentaje de especies para cada familia de reptiles en Mauritania.

\begin{tabular}{lcc}
\hline Family & Number of species & $\mathbf{\%}$ \\
\hline Colubridae & 14 & 16,3 \\
Lacertidae & 13 & 15,1 \\
Gekkonidae & 10 & 11,6 \\
Agamidae & 7 & 8,1 \\
Scincidae & 6 & 7,0 \\
Viperidae & 6 & 7,0 \\
Cheloniidae & 5 & 5,8 \\
Leptotyphlopidae & 3 & 3,5 \\
Elapidae & 3 & 3,5 \\
Varanidae & 3 & 3,5 \\
Atractaspididae & 2 & 2,3 \\
Chamaleonidae & 2 & 2,3 \\
Crocodylidae & 2 & 2,3 \\
Pelomedusidae & 2 & 2,3 \\
Trionychidae & 2 & 2,3 \\
Typhlopidae & 1 & 1,2 \\
Boidae & 1 & 1,2 \\
Dermochelyidae & 1 & 1,2 \\
Emydidae & 1 & 1,2 \\
Pythonidae & 1 & 1,2 \\
Testudinidae & 1 & 1,2 \\
\hline TOTAL & $\mathbf{8 6}$ & $\mathbf{1 0 0}$ \\
\hline & &
\end{tabular}

Bell, 1825; U. occidentalis Mateo, Geniez, LópezJurado \& Bons, 1998; Ptyodactylus oudrii Lataste, 1880; P. ragazzi (Andersson, 1898); Saurodactylus brosseti Bons \& Pasteur, 1957; Tarentola boehmei Joger, 1984; Sphenops boulengeri (Anderson, 1896); Macrovipera mauritanica (Gray, 1849); Naja haje (Linnaeus, 1758); b) Sahelian and Afrotropical species: Agama paragama Grandison, 1956; A. sankaranica Chabanaud, 1918; A. weiholzi Wettstein, 1932; Hemitheconyx caudicinctus (Duméril, 1851); Mabuya quinquetaeniata (Sternfeld, 1917); Dromophis praeornatus (Schlegel, 1837); Lycophidion albomaculatum Steindachner 1870; Philothamnus irregularis (Leach, 1819); Psammophis rukwae Spawls, 1983; Typhlops lineolatus (Jan, 1864); T. punctatus (Leach, 1819); Echis jogeri Cherlin, 1990; Kinixys belliana (Lataste, 1886); c) introduced species: Hemidactylus turcicus (Linnaeus, 1758) and Tarentola mauritanica (Linnaeus, 1758).
In summary, more than one hundred reptiles may be present in Mauritania. These data confer on Mauritania a good position in reptile diversity. For example, Joger \& Lambert (1997) reported 107 species for Mali, and 48 have been cited for Western Sahara (Geniez et al., 2004). However, we are far from having a complete panorama of the reptile diversity in Mauritania. Most records come from a handful of scattered localities and vast areas remain unexplored. For example, huge Saharan areas in the northern and northeastern parts of the country, and great part of the Sahel savannas in the south, lack any record of reptiles. Moreover, these savannas are highly endangered due to intense wood harvesting (mainly for firewood) and intense agropastoral use. These factors, together with long droughts have quickly transformed the environment. Therefore, most efforts should be oriented toward the study of diversity in the Sahel. The study of isolated wetlands in the Sahara, occupied by a mixture of Saharan and relict Sahelian elements, is also of high relevance if we are to understand the desert/savannah dynamics driven by climatic changes.

\section{ACKNOWLEDGEMENTS}

Fieldwork was funded by the Spanish International Cooperation Agency (AECI), Asociación Amigos de Doñana and L'Homme et la Nature: Mauritanie. I am specially indebted to Javier Castroviejo for encouraging my fieldwork in Mauritania. To E. Ávila, S. Castroviejo-Fischer, J. De Maille, M. Deida, M. Fadel, R. García-Romo, C. Carballo, J. PérezMarín, A. Quintana, M. Urcera and E. Mohamed-Saleh for their help in Mauritania. W. Böhme, P. Geniez, J. A. Mateo, H. Nickel, T. Wilms, Van Vallach and J. F. Trape provided information and/or literature. To W. Böhme, Ignacio De la Riva and an anonymous referee for their constructive criticisms on a previous draft of the manuscript. To Paul Bloor for improving the English.

\section{References}

ANGEL, F., 1933. Les serpents de l'Afrique occidentale francaise. Bulletin du Comité d'études Historiques et Scientifiques de l'Afrique Occidentale Française, 15: 613-858.

Angel, F., 1938. Liste des Reptiles de Mauritanie recueillis par la mission d'etudes de la Biologie des Acridiens en 1936 et 1937. Description d'une sousespéce nouvelle d'Eryx muelleri. Bulletin $d u$ Muséum National d'Historie Naturelle, $2^{\text {éme }}$ sèrie, 910: 485-487.

Angel, F., 1939. Deuxième liste des reptiles du Rio de Oro et de Mauritanie recueillis par la Mission d'études de la biologie des Acridiens (1937-1938). 
Bulletin du Muséum National d'Historie Naturelle, $2^{\text {éme }}$ sèrie, 11(1): 49-50.

Angel, F. \& Lothe, H., 1938. Reptiles et amphibiens du Sahara central et du Soudan. Bulletin du Comité d'études Historiques et Scientifiques de l'Afrique Occidentale Française, 21: 345-384.

Arnold, E. N. \& Leviton, A. E., 1977. A revision of the lizard genus Scincus (Reptilia: Scincidae). Bulletin of the British Museum (Natural History) Zoology, 31(5): 187-248.

Arvy, C. \& DiA, A. T., 1997. Donées sur les tortues marines et sur la tortue terrestre du littoral mauritanien. In P. Colas (ed.). Environnement et littoral mauritanien. Actes du colloque. CIRAD, Montpellier, Collections «Colloques»: 101-104.

Arvy, C., Maigret, J., Tijane, D. A. \& Colas, F., 1996. Observation des carapaces de tortues marines dans les villages cotiers de la grande plage mauritanienne (Cap Timirist-Frontière senégalaise). Bulletin de la Société Herpétologique Francaise, 79: 5-13.

Arvy, C., Marissal, N., Dia, A. T. \& Colas, F., 1997. Observations sur la répartition et les dimensions de Geochelone sulcata (Cryptodyra, Testudinidae) en Mauritanie Occidentale. Bulletin de la Société Herpétologique Francaise, 81: 11-20.

Aymerich, M., Bobrof-Aymerich, E. \& Geniez, P., 2004. Neufunde der seltenen Weißbäuchigen Sandrasselotter Echis leucogaster Roman, 1972 in Marokko. Herpetozoa, 16(3/4): 157-162.

Behra, O., 1994. Crocodiles on the desert's doorstep. Crocodile Specialist Group Newsletter, 13: 4-5.

BöHme, W., 1978. Zur herpetofaunistik des Senegal. Bonner zoologische Beiträge, 29(4): 360-417.

BöHME, W., 2000. Die wechselvolle Geschichte der Sahara: Untersuchung von Reptilienzönosen entlang eines westsaharischen Transektes, mit einem überraschenden Fund im Südosten Mauritaniens. Tier und Museum, 7(1): 11-21.

BöHME, W., 2003. Checklist of the living monitor lizards of the world (family Varanidae). Zoologische Verhandelingen, 341: 3-43.

Böhme, W., Wilms, T., Nickel, H. \& Merz, M., 2001. Bericht über einer herpetoogische Forschungsreise in die westliche Sahara und ihre Randgebiete. Zeitschrift Des Kölner Zoo, 44(3): 117-131.

Bons, J. \& Geniez, P., 1996. Anfibios y reptiles de Marruecos (Incluido Sáhara Occidental). Atlas biogeográfico. AHE. Barcelona. 320 p.

BötTGER, C. R., 1921. Meine Exkursion zur spanischen Kolonie Rio de Oro in Westafrika. Bericht Senckenberg Naturforschung Geselschaft, 51: 72-84.

Boulenger, G. A., 1919. A list of the snakes of West Africa from Mauritania to French Congo. Proceedings of the Zoological Society of London, 1919: 267-298.
Boulenger, G. A., 1921. Monograph of the Lacertidae. Vol II. Johnson Reprint Corporation. London. $451 \mathrm{pp.}$

BRito, J. C., 2003. Observations of amphibians and reptiles from north and west Africa - Morocco, Mauritania and Senegal. Boletín de la Asociación Herpetológica Española, 14(1-2): 2-6.

Chabanaud, P., 1916. Description d'un serpent nouveau de Mauritanie saharienne. Bulletin du Muséum National d'Historie Naturelle, Série Zoologique, 1916(2): 77-78.

Chabanaud, P., 1917. Enumération des reptiles no encore étudiés de l'Afrique occidentale, appartenant aux collections du Muséum, avec la description des espéces nouvelles. Bulletin du Muséum National d'Historie Naturelle, Série Zoologique, 1917(2): 83-105.

Chabanaud, P., 1924. Reptile recuillis par M. Th. Monod en Mauritanie et aux îles du Cap Vert. Bulletin $d u$ Muséum National d'Historie Naturelle, 1924: 54-56.

Chippaux, J. P., 2001. Les serpents d'Afrique occidentale et centrale. Éditions de l'IRD. Collection Faune et Flore tropicales. Paris. 202 pp.

Cissé, M. \& Karns, D. R., 1978. Les sauriens de Sénégal. Bulletin de l'Institut Français d'Afrique Noire, 10: 144-211.

Crochet, P. A., Geniez, P. \& Ineich, I., 2003. A multivariate analysis of the Acanthodactylus scutellatus group (Squamata: Lacertidae): systematics and biogeographical implications. Zoological Journal of the Linnean Society of London, 137(1): 117-155.

DeKeyser, P. L. \& Villiers A., 1956. Contribution a l'étude du peuplement de la Mauritanie. Notations ecologiques et biogeographiques sur la faune de l'Adrar. Mémoires de l'Institut Français d'Afrique Noire, 44: 1-222, $25 \mathrm{pl}$.

Donaire, D., Mateo, J. A., Hasi, M. \& Geniez, P., 2000. Nuevos datos sobre la fauna reptiliana de la Hamada de Tindouf (Argelia). Boletín de la Asociación Herpetológica Española, 11(1):8-12.

EMBL RePtile DataBASE, 2006. http://www.embl-heidelberg.de/ uetz/LivingReptiles.html. (Accessed March 2006).

Fuchs, K., Mertens, R. \& Wermuth, H., 1974. Zum status vom Crocodylus cataphractus und Osteolaemus tetraspis. Stuttgarter Beiträge zur Naturkunde, Serie A (Biologie), 266: 1-8.

Geniez, P. \& FoucART, A., 1995. Un nouvel Acanthodactyle en Algérie: Acanthodactylus taghitensis n. sp. (Reptilia, Sauria, Lacertidae). Bulletin du Muséum National d'Historie Naturelle, $4^{\text {éme }}$ série, 17(1-2): 3-9.

Geniez, P. \& ARnold, E. N., 2006. A new species of Semaphore gecko Pristurus (Squamata: Gekkonidae) from Mauretania, represents a $4700 \mathrm{~km}$ range extension for genus. Zootaxa, 1317: 57-68. 
Geniez, P. \& Luch, P., 2004. New data on fringe-toed lizards of the Acanthodactylus scutellatus group in Mauritania. Herpetozoa, 17(1/2): 88-89.

Geniez, P., Mateo, J. A. \& Bons, J., 2000. A checklist of the amphibians and reptiles of Western Sahara (Amphibia, Reptilia). Herpetozoa, 13(3/4): 149-163.

Geniez, P., Mateo, J. A., Geniez, M. \& Pether, J., 2004. The Amphibians and Reptiles of Western Sahara. An atlas and field guide. Chimaira. Frankfurt an Maim. $229 \mathrm{pp}$.

Grandison, A. C. G., 1956. On a collection of lizards from West Africa. Bulletin de l'Institut Français d'Afrique Noire (A), 18: 224-245.

Grandison, A. G. C., 1961. Prelimiary notes on the taxonomy of Tarentola annularis and T. ephippiata (Sauria: Gekkonidae). Zoologische Mededelingen, 38(1): 1-14.

Hahn, D. E. \& Wallach, V., 1998. Comments on the systematics of Old World Leptotyphlops (Serpentes: Leptotyphlopidae), with description of a new species. Hamadryad, 23: 50-62.

Hasi, M., López-Jurado, L. F., Mateo, J. A., SaintANDRIEUX, J. P. \& Geniez, P., 1997. Nouvelles observations herpetologiques au Sahara Occidental, 3. Bulletin de la Société Herpétologique Française, 84: 33-38.

Heimes, P., 1987. Beitrag zur Systematik der Fächerfinger (Sauria: Gekkonidae: Ptyodactylus). Salamandra, 23(4): 212-235.

Hughes, B., 1983. African snake faunas. Bonner zoologishe Beiträge, 34: 311-356.

IGN, 1993. Mauritanie. Carte Générale au 1:2500000. Institut Géographique National. Paris.

INEICH, I., 1997. Les amphibiens et reptiles du littoral mauritanien. In: P. Colas (ed.). Environnement et littoral mauritanien. Actes du colloque. CIRAD, Montpellier, Collections «Colloques»: 93-99.

Ineich, I., Dia, A. T., Alphaly, B. \& Colas, F., 1998. Gechoniza chazaliae. A correction. Herpetological Review, 29(1): 51.

Ineich, I., Dia, A. T., Alphaly, B., Girard, F. \& Colas, F., 1997. Geckonia chazaliae. Maximum size. Herpetological Review, 28(4): 202-203.

Iverson, J. B., 1992. A Revised Checklist with Distribution Maps of the Turtles of the World. Privately printed. Paust Printing. Richmond. 363 pp.

JoGER, U., 1979. Zur ökologie und Verbreitung wenig bekannter Agamen Westafrikas (Reptilia: Sauria: Agamidae). Salamandra, 15(1): 31-52.

Joger, U., 1980. Eine neue Art der Gattung Tarentola (Reptilia: Sauria: Gekkonidae) aus Westafrika. Amphibia-Reptilia, 1: 137-147.

Joger, U., 1981. Zur Herpetofaunistik Westafrikas. Bonner zoologische Beiträge, 32(3/4): 297-340.
Joger, U., 1984. Taxonomische Revision der Gattung Tarentola (Reptilia: Gekkonidae). Bonner zoologische Beiträge, 35(1-3): 129-174.

Joger, U. \& Lambert, M. R. K., 1996. Analysis of the herpetofauna of the Republic of Mali, I. Annotated inventory, with description of a new Uromastyx (Sauria: Agamidae). Journal of African Zoology, 110(1): 21-51.

Joger, U. \& LAmbert, M. R. K., 1997. Analysis of the herpetofauna of the Republic of Mali, II. Species diversity and biogeographical assemblages. In: $\mathrm{H}$. Ulrich (ed.). Tropical biodiversity and systematics. Proceedings of the International Symposium on Biodiversity and Systematics in Tropical Ecosystems, Bonn, 1994. Zoologisches Forschunginstitut und Museum Alexander Koening. Bonn: 189-202.

JolEAUD, L., 1933. Études de Gèographie zoologuique sur la Berbèrie. Bulletin de la Sociètè Zoologique de France, 58: 397-404.

Jullien, R. \& Petter, F., 1970. La faune du gisement d'Akjoujt (Mauritanie). Bulletin Muséum National

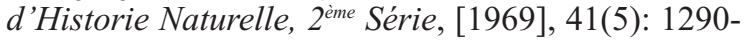
1291.

LAmbert, M. R. K., 1984. Amphibians and Reptiles. In: J. L. Cloudsley-Thompson. (ed.). Sahara Desert. Key Environments. Pergamon Press. Oxford: 205-227.

Lambert, M. R. K. \& Mullié, W. C., 1998. Sexual dichromatism of Agama boulengeri observed in southern Mauritania. British Herpetological Society Bulletin, 65: 42-44.

Le Berre M., 1989. Faune du Sahara. I. PoissonsAmphibiens-Reptiles. Lechevalier-R. Chabaud. Paris. 332 pp.

Le Toquin, A., Galmel, E. \& Trotignon, J., 1980. Morphologie, croissance individuelle et dynamique des populations de la tortue verte (Chelonia mydas L.) au Banc d'Arguin (Republique Islamique de Mauritanie). Revue de Écologie Terre et Vie, 34: 271-302.

LOVERIDGE, A., 1941. Revision of the African Snakes of the genera Dromophis and Psammophis. Bulletin of the Museum of Comparative Zoology, 87: 1-69.

LOVERIDGE, A. \& Williams, E. E., 1957. Revision of the African Tortoises and turtles of the suborder Cryptodira. Bulletin of the Museum of Comparative Zoology, 115(6): 163-557.

MAHÉ, E., 1985. Contribution à l'étude scientifique de la région du Banc d'Arguin (littoral mauritanien). Peuplements avifaunistiques. Thèse de Doctorat d'Université (Écologie). Montpellier. 576 pp.

MAigret, J., 1975. Notes et informations: les tortues de mer du banc d'Arguin. Bulletin du Laboratoire des Pêches de Nouâdhibou (Mauritanie), 4: 118-119.

Maigret, J., 1983. Répartition des tortues de mer sur les côtes ouest-africaines. Bulletin de la Société Herpétologique Française, 28: 22-34. 
Mateo, J. A., Geniez, P., López-Jurado, L. F. \& Bons, J., 1998. Chrorological analysis and morphological variations of Saurians of the genus Uromastyx (Reptilia, Agamidae) in western Sahara. Description of two new taxa. Revista Española de Herpetología, 12: 97-109.

MAYr, E., 1942. Systematics and the origin of species from the viewpoint of a zoologist. 3rd Ed. 1999. Harvard University Press. Cambridge. 334 pp.

Monod, T., 1921. Remarques biologiques sur le Sahara. Revue générale des sciences, 42: 609-616.

Monod, T., 1928. Une traversée de la Mauritanie occidentale. Revue de géographie physique et de géologie dynamique, I, 1: 10.

Moquard, F., 1895. Note sur quelques reptiles du Cap Blanc. Bulletin du Muséum d'Histoire Naturelle, 1: 310-312.

NiCKEL, H., 2003. Ökologische Untersuchungen zur Wirbeltierfauna in südöstilichen Mauretanien. Zwei Fallstudien unter besonderer Berücksichtigung der Krokodile. GTZ. Echborn. 89 pp.

Padial, J. M., 2003. On the presence of Python sebae Gmelin, 1788 (Ophidia: Pythonidae) in Mauritania. Herpetological Bulletin, 84: 30-31.

Padial, J. M., 2005. A new species of Agama (Sauria: Agamidae) from Mauritania. Herpetological Journal, 15: 27-35

Padial, J. M., Castroviejo-Fisher, S. Quintana, A. Z., Avila, E., Marín, J. \& Castroviejo, J., 2002. Notas de distribución de reptiles para el noroeste de África. Boletín de la. Asociación Herpetológica Española, 13(1-2): 2-5.

Padial, J. M. \& De LA Riva, I., 2004. Annotated checklist of the amphibians of Mauritania (West Africa). Revista Española de Herpetología, 18: 89-99.

PADial, J. M. \& DE LA Riva, I. 2006. Taxonomic Inflation and the Stability of Species Lists: the Perils of Ostrich's Behavior. Systematic Biology, 55(5): 859-867.

Pellegrin, J., 1910. Mission en Mauritanie occidentale III: partie zoologique. Reptiles. Actes de la Société Linnéenne de Bordeaux, 64: 21-25.

Pellegrin, J., 1911. Les vertèbrès aquatiques du Sahara. Compte Rendu de l'Académie des Sciences, 153: 972-974.

Pleguezuelos, J. M., Fariña, B., Mateo, J. A. \& Geniez, P., 2005. Nuevos datos sobre los anfibios y reptiles de Mauritania y el Sáhara Occidental. Boletín de la Asociación Herpetológica Española, 15(2): 76-80.

Roux-Estève, R., 1974. Révision systématique des Typhlopidae d'Afrique: Reptilia-Serpentes. Mémoires du Muséum national d'histoire naturelle, Paris, Sér. 2, 87A: 1-313.
SAlVAdOR, A., 1982. A revision of the lizards of the genus Acanthodactylus (Sauria: Lacertidae). Bonner zoologische Monographien, 16: 1-167.

SchÄтtI, B., 1986. Morphologie und Systematik von Coluber algirus und C. hippocrepis (Reptilia: Colubridae). Bonner zoologische Beiträge, 37(4): 281-293.

Schleich, H. H., Kästle, W. \& Kabisch, K., 1996. Amphibians and Reptiles of North Africa. Koeltz Scientific Books. Königstein. 627 pp.

Schmiz, A., Mansfeld, P., Hekkala, E., Shine, T., Nickel, H., Amato, G. \& BöHme, W., 2003. Molecular evidence for species level divergence in African Nile Crocodiles Crocodylus niloticus (Laurenti, 1786). Comptes Rendus Palevol, 2: 703-712.

Shine, T., Böhme, W., Nickel, H., Thies, D. F. \& Wilms, T., 2001. Rediscovery of relict popuations of the Nile crocodile Crocodylus niloticus in south-eastern Mauritania, with observations on their natural history. Oryx, 35(3): 260-262.

Spatz, P., 1930. Reisen aus den Jahren 1928 und 1929 nach dem Senegal und Mauritanien. Zeitschrift für Saugetierkunde, 5: 7-13.

STAudingER, P., 1929. Krokodile in der Inner-Sahara und in Mauritanien. Sitzungberichte der Gesellschaft Naturforschender Freunde Zu Berlin, 1992: 141142.

Thorpe, R. S. \& MCCARTHY, C. J., 1978. A preliminary study using multivariate analysis of a species complex of African House snake (Boaedon fuliginosus). Journal of Zoology, London, 184: 489-506.

TrAPE, J. F., 2002. Note sur le statut et la repartition de quelques Leptotyohlopidés (Serpentes; Scolecophidia) du Sahara et des savanes d'Afrique de l'Ouest. Bulletin de la Société Herpétologique de France, 102: 49-62.

Trape, J. F. \& MANÉ, Y., 2002. Les serpents du Sénégal: liste commentée des espèces. Bulletin de la Société de pathologie exotique, 95(3): 148-150.

Villiers, A., 1950. Contribution a l'étude du peuplement de la Mauritanie. Ophidiens. Bulletin de l'Institut Français d'Afrique Noire, 12(4): 984-998.

VILLIERS, A., 1951. La collection de Serpents de l'IFAN (Acquisitions 1950). Bulletin de l'Institut Français d'Afrique Noire, sér. A, 13(4): 1234-1247.

VILLIERS, A., 1954. La collection de Serpents de l'IFAN (Acquisitions 1953). Bulletin de l'Institut Français d'Afrique Noire, sér. A, 16(4): 813-836.

Villiers, A., 1956. La collection de Serpents de l'I.F.A.N. (Acquisitions 1954-1955). Bulletin de l'Institut Français d'Afrique Noire, sèr. A, 18(3): 877-883.

Villiers, A., 1958. Tortues et crocodiles de l'Afrique Noire Française. Initiation Africaines, l'Institut Français d'Afrique Noire, 15: 1-354. 
VILLIERS, A., 1975. Les serpents de l'ouest africain. $3^{\mathrm{ème}}$ éd.. Initiations études africaines. II. Dakar. 195 pp.

Wallach, V., 2002. Typhlops etheridgei, a new species of African blindsnake in the Typhlops vermicularis species group from Mauritania (Serpentes: Typhlopidae). Hamadryad, 27(1): 108-122.

WCMC, 2006. http://www.unep-wcmc.org/. (Accessed March 2006).

Welch, K. R. G., 1982. Herpetology of Africa: a checklist and bibliography of the orders Amphisbaenia, Sauria, and Serpentes. R.E. Krieger Publishing. Malabar. x +293 pp.
WiLms, T. \& BöHME, W., 2001. Revision der Uromastyx acanthinura-Artengruppe, mit Beschreibung einer neuen Art aus der Zentralsahara (Reptilia: Sauria: Agamidae). Zoologische Abhandlungen Staatliches Museum für Tierkunde Dresden, 51(8): 73-104.
Recibido, 10-III-2006

Aceptado, 20-X-2006

Publicado, 29-XII-2006 
Appendix.- Reptile localities of Mauritania.

Apéndice.- Localidades de los reptiles Mauritania.

\begin{tabular}{|c|c|c|c|c|c|}
\hline LOCALITIES & COORDINATES & $\begin{array}{l}\text { Bahie du Lévrier } \\
\text { Banc d'Arguin, Nation }\end{array}$ & $\begin{array}{l}20^{\circ} 50^{\prime} \mathrm{N}, 17^{\circ} 24^{\prime} \mathrm{W} \\
19^{\circ} 26^{\prime} \mathrm{N}, 16^{\circ} 19^{\prime} \mathrm{W}\end{array}$ & $\begin{array}{l}\text { Echekraf } \\
\text { El Mhaïjrât }\end{array}$ & $\begin{array}{r}19^{\circ} 36,450^{\prime} \mathrm{N}, 16^{\circ} 0,821^{\prime} \mathrm{W} \\
19^{\circ} 01.64^{\prime} \mathrm{N}, 16^{\circ} 13.92^{\prime} \mathrm{W}\end{array}$ \\
\hline \multicolumn{2}{|l|}{ Adrar } & Birguendouz & $21^{\circ} 36^{\prime} 34.3^{\prime \prime N}, 16^{\circ} 27^{\prime} 59.7^{\prime \prime} \mathrm{W}$ & Jraiff & $19^{\circ} 15.98^{\prime} \mathrm{N}, 16^{\circ} 28.42^{\prime} \mathrm{W}$ \\
\hline$\overline{\text { Aggui }}$ & $21^{\circ} 10^{\prime} \mathrm{N}, 13^{\circ} 07^{\prime} \mathrm{W}$ & Boû Lanouâr & $21^{\circ} 16.120^{\prime} \mathrm{N}, 16^{\circ} 31.950^{\prime} \mathrm{W}$ & Tenbrahim & $19^{\circ} 37.762^{\prime} \mathrm{N}, 15^{\circ} 56.764 \mathrm{~W}$ \\
\hline & $20^{\circ} 32^{\prime} \mathrm{N}, 12^{\circ} 48^{\prime} \mathrm{W}$ & Boû Lânoir-Inâl, betw & veen & Tijirît & $19^{\circ} 38,777^{\prime} \mathrm{N}, 16^{\circ} 0,821^{\prime} \mathrm{W}$ \\
\hline $\begin{array}{l}\text { Amogjar pass } \\
\text { Atar }\end{array}$ & $20^{\circ} 31^{\prime} \mathrm{N}, 13^{\circ} 03^{\prime} \mathrm{W}$ & \multicolumn{2}{|c|}{$21^{\circ} 17.038^{\prime} \mathrm{N}, 15^{\circ} 08.371^{\prime} \mathrm{W}$} & & \\
\hline Ain Savra & $19^{\circ} 32.416^{\prime} \mathrm{N}, 12^{\circ} 06.829^{\prime} \mathrm{W}$ & Cap Arguin & $20^{\circ} 33^{\prime} 32.2^{\prime \prime} \mathrm{N}, 16^{\circ} 31^{\prime} 46.2^{\prime \prime}$ & \multicolumn{2}{|l|}{ Nouakchott } \\
\hline Azougui & $20^{\circ} 30^{\prime} \mathrm{N}, 13^{\circ} 10^{\prime} \mathrm{W}$ & Cap Blanc & $20^{\circ} 54^{\prime} \mathrm{N}, 17^{\circ} 04^{\prime} \mathrm{W}$ & Nouakchott & $18^{\circ} 4^{\prime} 51.1^{\prime \prime} \mathrm{N}, 15^{\circ} 58^{\prime} 55.55^{\prime \prime} \mathrm{W}$ \\
\hline Ben Amira & $21^{\circ} 13.765^{\prime} \mathrm{N}, 13^{\circ} 40.329^{\prime} \mathrm{W}$ & Cap Timirist & $19^{\circ} 23^{\prime} \mathrm{N}, 16^{\circ} 32^{\prime} \mathrm{W}$ & \multirow[t]{2}{*}{ Port of Nouakchott } & \multirow[t]{2}{*}{$18^{\circ} 06.30^{\prime} \mathrm{N}, 16^{\circ} 01.55^{\prime} \mathrm{W}$} \\
\hline Châr area & $21^{\circ} 18^{\prime} \mathrm{N}, 11^{\circ} 00^{\prime} \mathrm{W}$ & El Aïouej & $21^{\circ} 15^{\prime} \mathrm{N}, 16^{\circ} 40^{\prime} \mathrm{W}$ & & \\
\hline Chinguetti & $20^{\circ} 27^{\prime} \mathrm{N}, 12^{\circ} 22^{\prime} \mathrm{W}$ & Inâl & $21^{\circ} 17.165 \mathrm{~N}, 14^{\circ} 59.720^{\prime} \mathrm{W}$ & \multicolumn{2}{|l|}{$\underline{\text { Tagant }}$} \\
\hline Oued Choûm & $21^{\circ} 17.050^{\prime} \mathrm{N}, 13^{\circ} 06.060^{\prime} \mathrm{W}$ & Iouîk & $19^{\circ} 53.055^{\prime} \mathrm{N} .16^{\circ} 17.696^{\prime} \mathrm{W}$ & $\overline{\text { Adâfer Meridional }}$ & $19^{\circ} 30^{\prime} \mathrm{N}, 10^{\circ} 00^{\prime} \mathrm{W}$ \\
\hline Dahr Chinguetti & $20^{\circ} 26.547^{\prime} \mathrm{N}, 12^{\circ} 49.407^{\prime} \mathrm{W}$ & La Güera & $20^{\circ} 49^{\prime} 51.2^{\prime \prime} \mathrm{N}, 17^{\circ} 5^{\prime} 14.9^{\prime \prime} \mathrm{W}$ & Atar-Tidjikja, between & $20^{\circ} 22.412^{\prime} \mathrm{N}, 12^{\circ} 45.625 \mathrm{~W}$ \\
\hline E of Ouadane & $20^{\circ} 59^{\prime} \mathrm{N}, 11^{\circ} 26^{\prime} \mathrm{W}$ & Mamghar & $19^{\circ} 21.631^{\prime} \mathrm{N}, 16^{\circ} 30.674^{\prime} \mathrm{W}$ & Guelta Fanar & $18^{\circ} 0.951^{\prime} \mathrm{N}, 12^{\circ} 10.498^{\prime} \mathrm{W}$ \\
\hline El Beyyed & $21^{\circ} 27^{\prime} \mathrm{N}, 11^{\circ} 29^{\prime} \mathrm{W}$ & Marzouba & $21^{\circ} 01.490^{\prime} \mathrm{N}, 16^{\circ} 29.290^{\prime} \mathrm{W}$ & Guelta Matmata & $17^{\circ} 53^{\prime} \mathrm{N}, 12^{\circ} 07^{\prime} \mathrm{W}$ \\
\hline El Gueldi & $20^{\circ} 57.783^{\prime} \mathrm{N}, 13^{\circ} 11.002^{\prime} \mathrm{W}$ & Nouâdhibou & $20^{\circ} 55.183^{\prime} \mathrm{N}, 17^{\circ} 02.714^{\prime} \mathrm{W}$ & Lekhcheb & $18^{\circ} 30.928^{\prime} \mathrm{N}, 10^{\circ} 0.769^{\prime} \mathrm{W}$ \\
\hline Ez Zerga & $20^{\circ} 22.412^{\prime} \mathrm{N}, 12^{\circ} 45.625^{\prime} \mathrm{W}$ & Oued ech Chibka & $20^{\circ} 36^{\prime} 5.2^{\prime \prime} \mathrm{N}, 16^{\circ} 5^{\prime} 53.2^{\prime \prime} \mathrm{W}$ & Tîchît & $18^{\circ} 26.380^{\prime} \mathrm{N}, 9^{\circ} 29.666^{\prime} \mathrm{W}$ \\
\hline Guelb er Richât & $21^{\circ} 03^{\prime} \mathrm{N}, 11^{\circ} 15^{\prime} \mathrm{W}$ & Oued Khatt Atoui & $20^{\circ} 04^{\prime} 12^{\prime \prime} \mathrm{N}, 15^{\circ} 58^{\prime} 48^{\prime \prime} \mathrm{W}$ & Tidjikja & $18^{\circ} 33^{\prime} \mathrm{N}, 11^{\circ} 25^{\prime} \mathrm{W}$ \\
\hline Guelta Hamdoun & $20^{\circ} 20^{\prime} \mathrm{N}, 13^{\circ} 30^{\prime} \mathrm{W}$ & Tafarit Cape & $20^{\circ} 07^{\prime} 50^{\prime \prime} \mathrm{N}, 16^{\circ} 15^{\prime} 41^{\prime \prime} \mathrm{W}$ & Zig & $18^{\circ} 34.487^{\prime} \mathrm{N}, 9^{\circ} 48.379^{\prime} \mathrm{W}$ \\
\hline Guelta Molomhar & $20^{\circ} 34^{\prime} \mathrm{N}, 13^{\circ} 07^{\prime} \mathrm{W}$ & Tanoudert & $20^{\circ} 20^{\prime} \mathrm{N}, 16^{\circ} 02^{\prime} \mathrm{W}$ & & \\
\hline Ijâfen area (BM 1975.1 & 398) $\quad 20^{\circ} 27.4^{\prime} \mathrm{N}, 8^{\circ} 48^{\prime} \mathrm{W}$ & Tidra Island & $19^{\circ} 35^{\prime} 95^{\prime \prime} \mathrm{N}, 16^{\circ} 25^{\prime} 97^{\prime \prime} \mathrm{W}$ & Tiris Zemmour & \\
\hline Iriji & $20^{\circ} 31^{\prime} \mathrm{N}, 13^{\circ} 03^{\prime} \mathrm{W}$ aprox. & Timirist Cape & $19^{\circ} 22^{\prime} 34.1^{\prime \prime} \mathrm{N}, 16^{\circ} 32^{\prime} 22.3^{\prime \prime} \mathrm{W}$ & & $21^{\circ} 47^{\prime} \mathrm{N}, 12^{\circ} 07^{\prime} \mathrm{W}$ \\
\hline Kanoal, Oued Séguelil & $20^{\circ} 18.216^{\prime} \mathrm{N}, 13^{\circ} 11.837^{\prime} \mathrm{W}$ & Tmeimichat & $21^{\circ} 14.281^{\prime} \mathrm{N}, 14^{\circ} 23.570^{\prime} \mathrm{W}$ & Bir Moghrein/Fort Tring & guet \\
\hline Legleitât & $19^{\circ} 59^{\prime} \mathrm{N}, 13^{\circ} 17^{\prime} \mathrm{W}$ & $50 \mathrm{~km}$ East of Boû & Lanouâr, track Nouadhibou- & 25 & $25^{\circ} 13^{\prime} 38.1^{\prime \prime} \mathrm{N}, 11^{\circ} 34^{\prime} 45.3^{\prime \prime} \mathrm{W}$ \\
\hline Ouadâne & $20^{\circ} 50^{\prime} \mathrm{N}, 11^{\circ} 36^{\prime} \mathrm{W}$ & Choum & $21^{\circ} 17^{\prime} \mathrm{N}, 16^{\circ} 01^{\prime} \mathrm{W}$ & Dayet el Am & $25^{\circ} 56^{\prime} \mathrm{N}, 8^{\circ} 25^{\prime} \mathrm{W}$ \\
\hline Ouarâne & $20^{\circ} 27.4^{\prime} \mathrm{N}, 8^{\circ} 48^{\prime} \mathrm{W}$ & & & Hasi el Fogra & $27^{\circ} 04^{\prime} \mathrm{N}, 8^{\circ} 21^{\prime} \mathrm{W}$ \\
\hline Oued Choûm & $21^{\circ} 22.6^{\prime} \mathrm{N}, 12^{\circ} 58.6^{\prime} \mathrm{W}$ & Gorgol & & Fdérik (Fort Gouraud) & $22^{\circ} 41.659^{\prime} \mathrm{N}, 12^{\circ} 42.912^{\prime} \mathrm{W}$ \\
\hline road Atar-Chinguetti vi & a Ebnou Pass & Kaedi & $16^{\circ} 09^{\prime} \mathrm{N}, 13^{\circ} 30^{\prime} \mathrm{W}$ & Northern Tagenzé, Ouan & n n'Namour \\
\hline & $20^{\circ} 33^{\prime} \mathrm{N}, 12^{\circ} 36^{\prime} \mathrm{W}$ & M'bout & $16^{\circ} 1^{\prime} 41.8^{\prime \prime} \mathrm{N}, 12^{\circ} 34^{\prime} 37.5^{\prime \prime} \mathrm{W}$ & & $21^{\circ} 22^{\prime} 51^{\prime \prime} \mathrm{N}, 11^{\circ} 24^{\prime} 36^{\prime \prime} \mathrm{W}$ \\
\hline Tacarât area (BM 1975 & $20^{\circ} 44.5^{\prime} \mathrm{N}, 10^{\circ} 30 \mathrm{~W}$ & & & Road N50 near the Sahar & ara Border $27^{\circ} 14^{\prime} \mathrm{N}, 8^{\circ} 25^{\prime} \mathrm{W}$ \\
\hline $\begin{array}{l}\text { lasiast, Ogla de lamar } \\
\text { Te-n-Zak pass, road } \mathrm{Ch}\end{array}$ & & $\frac{\text { Guidimaka }}{\text { Lake Houi }}$ & & Toûâjil $\quad 22$ & $22^{\circ} 10^{\prime} 17.2^{\prime \prime} \mathrm{N}, 12^{\circ} 40^{\prime} 18.2^{\prime \prime} \mathrm{W}$ \\
\hline $\begin{array}{l}\text { Terjît } \\
\text { Ter-Zak pass, road }\end{array}$ & $\begin{aligned} \text { oum-Atar } 20^{\circ} \\
20^{\circ} 15.578^{\prime} \mathrm{N}\end{aligned}$ & Lake Hou1 & 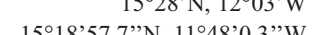 & Tourîne area & $22^{\circ} 25^{\prime} \mathrm{N}, 11^{\circ} 52^{\prime} \mathrm{W}$ \\
\hline $\begin{array}{l}\text { lerjlt } \\
\text { Tintane }\end{array}$ & $\begin{array}{r}15.578^{\prime} \mathrm{N}, \\
19^{\circ} 50\end{array}$ & Bouli & 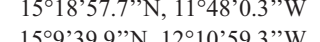 & Zemmour & $25^{\circ} 3^{\prime} 50.4^{\prime \prime} \mathrm{N}, 11^{\circ} 57^{\prime} 28.1^{\prime \prime} \mathrm{W}$ \\
\hline Zerga mountains, ab & $\mathrm{n}$ WSW of Ch & Sellbab1 & $159939.9^{\prime \prime} \mathrm{N}, 12$ & Zouérat & $22^{\circ} 44.00^{\prime} \mathrm{N}, 12^{\circ} 28.00^{\prime} \mathrm{W}$ \\
\hline & $20^{\circ} 19^{\prime} 11.6^{\prime \prime} \mathrm{N}, 12^{\circ} 40^{\prime} 52^{\prime \prime} \mathrm{W}$ & Hodh Ech Chargui & & Zouérat-Bir Moghrein, b & between $24^{\circ} 46^{\prime} \mathrm{N}, 11^{\circ} 46^{\prime} \mathrm{W}$ \\
\hline $44 \mathrm{~km}$ from Chinguett & on the road to Adrar, $19 \mathrm{~km}$ & Adel Bagrou & $8^{\circ} 02^{\prime} \mathrm{N}, 15^{\circ} 55^{\prime} \mathrm{W}$ & & \\
\hline before the Nouatil $\mathrm{P}$ & & Dendaré & $16^{\circ} 17^{\prime} 12.1^{\prime \prime} \mathrm{N}, 6^{\circ} 41^{\prime} 12.8^{\prime \prime} \mathrm{W}$ & & \\
\hline & $20^{\circ} 32^{\prime} 45.2^{\prime \prime} \mathrm{N}, 12^{\circ} 17^{\prime} 22.2^{\prime \prime} \mathrm{E}$ & Mahmûdé Lake & $16^{\circ} 29.969 \mathrm{~N}, 7^{\circ} 42.911^{\prime} \mathrm{W}$ & $50 \mathrm{~km} \mathrm{NE} \mathrm{Nou}$ & 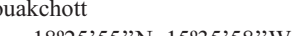 \\
\hline $30 \mathrm{~km} \mathrm{E}$ of Atar, road & Atar-Chinguetti via Amogjar & Oualâta & $17^{\circ} 17^{\prime} 47.5^{\prime \prime} \mathrm{N}, 7^{\circ} 1^{\prime} 24.3^{\prime \prime} \mathrm{W}$ & & $18^{\circ} 25^{\prime} 55^{\prime \prime} \mathrm{N}, 15^{\circ} 35^{\prime} 58^{\prime \prime} \mathrm{W}$ \\
\hline pass & $20^{\circ} 34^{\prime} \mathrm{N}, 12^{\circ} 52^{\prime} \mathrm{W}$ & Timbedgha & $16^{\circ} 14^{\prime} 41.4^{\prime \prime} \mathrm{N}, 8^{\circ} 10^{\prime} 5.5^{\prime \prime} \mathrm{W}$ & Aleib Ataf & $18^{\circ} 45^{\prime} 31$ '”N, 16 6'29'”W \\
\hline $50 \mathrm{~km} \mathrm{~S}$ of Choum, ro & d Choum-Atar & & & Bafrechié & $6^{\circ} 50^{\prime} 50.9^{\prime \prime} \mathrm{N}, 15^{\circ} 29^{\prime} 46.5^{\prime \prime} \mathrm{W}$ \\
\hline & $21^{\circ} 00^{\prime} \mathrm{N}, 13^{\circ} 09^{\prime} \mathrm{W}$ & Hodh EI Gharbi & & Blaouakh & $18^{\circ} 30.98^{\prime} \mathrm{N}, 16^{\circ} 04.45^{\prime} \mathrm{W}$ \\
\hline $65 \mathrm{Km} \mathrm{S}$ of Atar & $20^{\circ} 15.077^{\prime} \mathrm{N}, 13^{\circ} 22.847^{\prime} \mathrm{W}$ & $\overline{\text { Ayoûn El Atroûs }}$ & $16^{\circ} 39^{\prime} 41^{\prime \prime} \mathrm{N}, 9^{\circ} 37^{\prime} 2.3^{\prime \prime} \mathrm{W}$ & Boutlimit & $17^{\circ} 32^{\prime} 41^{\prime \prime} \mathrm{N}, 14^{\circ} 41^{\prime} 56^{\prime \prime} \mathrm{W}$ \\
\hline & & Chlim & $9^{\circ} 05^{\prime} \mathrm{N}, 16^{\circ} 42^{\prime} \mathrm{W}$ & Campament 28 & $17^{\circ} 41.55^{\prime} \mathrm{N}, 16^{\circ} 02.61^{\prime} \mathrm{W}$ \\
\hline$\underline{\text { Assaba }}$ & & Foulania & $15^{\circ} 31^{\prime} \mathrm{N}, 9^{\circ} 49^{\prime} \mathrm{W}$ & Campament 65 & $17^{\circ} 31.35^{\prime} \mathrm{N} 16^{\circ} 04.93{ }^{\prime} \mathrm{W}$ \\
\hline Aïn El Ghaire & $17^{\circ} 11.70^{\prime} \mathrm{N}, 12^{\circ} 14.92^{\prime} \mathrm{W}$ & Oumm El Khez & $17^{\circ} 3.705^{\prime} \mathrm{N}, 10^{\circ} 57.278^{\prime} \mathrm{W}$ & Chott Boul & $16^{\circ} 38^{\prime} 00^{\prime \prime} \mathrm{N}, 16^{\circ} 26^{\prime} 40^{\prime \prime} \mathrm{W}$ \\
\hline Bou Bleï’îne & $17^{\circ} 07.564^{\prime} \mathrm{N}, 10^{\circ}$ & Tâmchekket & $17^{\circ} 14^{\prime} 50.6 " \mathrm{~N}, 10^{\circ} 40^{\prime} 23^{\prime \prime} \mathrm{W}$ & Dara & $16^{\circ} 36.323^{\prime} \mathrm{N}, 16^{\circ} 7.969^{\prime} \mathrm{W}$ \\
\hline Bougari & $16^{\circ} 32.034^{\prime} \mathrm{N}, 10^{\circ} 47.892^{\prime} \mathrm{W}$ & Timbedgha-Ayoûn E & 1 Atroûs, between & Dar es Salam & $16^{\circ} 36.158^{\prime} \mathrm{N}, 16^{\circ} 05.491 \mathrm{~W}$ \\
\hline Foum El Cherat & $16^{\circ} 30^{\prime} 35^{\prime \prime} \mathrm{N}, 10^{\circ} 49^{\prime} 3$ '”W & & $16^{\circ} 26.890^{\prime} \mathrm{N}, 9^{\circ} 14.690^{\prime} \mathrm{W}$ & Diama-Rosso, road & $16^{\circ} 33^{\prime} \mathrm{N}, 16^{\circ} 05^{\prime} \mathrm{W}$ \\
\hline Guelta Metraucha & $16^{\circ} 33^{\prime} 48^{\prime \prime} \mathrm{N}, 10^{\circ} 45^{\prime} 17^{\prime \prime} \mathrm{W}$ & Tintâne & $16^{\circ} 23.522^{\prime} \mathrm{N}, 10^{\circ} 10.405^{\prime} \mathrm{W}$ & El Hazra & $18^{\circ} 58^{\prime} 43^{\prime \prime} \mathrm{N}, 16^{\circ} 12^{\prime} 07^{\prime \prime} \mathrm{W}$ \\
\hline Guelta Oumm Lebare & $16^{\circ} 29.472^{\prime} \mathrm{N}, 10^{\circ} 49.822 \mathrm{~W}$ & Zoueina & $15^{\circ} 42.689^{\prime} \mathrm{N}, 9^{\circ} 39.906^{\prime} \mathrm{W}$ & El Msid & $18^{\circ} 41.18^{\prime} \mathrm{N}, 16^{\circ} 06.09^{\prime} \mathrm{W}$ \\
\hline Kiffa & $16^{\circ} 36.850^{\prime} \mathrm{N}, 11^{\circ} 24.930^{\prime} \mathrm{W}$ & & & Grand Plage & $17^{\circ} 32^{\prime} \mathrm{N}, 16^{\circ} 04^{\prime} \mathrm{W}$ \\
\hline Louths & $17^{\circ} 14.40^{\prime} \mathrm{N}, 12^{\circ} 06.04^{\prime} \mathrm{W}$ & $\underline{\text { Inchiri }}$ & & Hasseï Gáboûn & $18^{\circ} 16^{\prime} 22^{\prime \prime} \mathrm{N}, 15^{\circ} 51^{\prime} 01^{\prime \prime} \mathrm{W}$ \\
\hline & & Agneïtîr & $19^{\circ} 21^{\prime} 3.5^{\prime \prime} \mathrm{N}, 16^{\circ} 15^{\prime} 25.3^{\prime \prime} \mathrm{W}$ & Keur Macène & $16,23^{\circ} \mathrm{N}, 16,55^{\circ} \mathrm{W}$ \\
\hline Brakna & & Agneïtir, Elb Aouarou & lâr, 30 km from Jreïf & Lekeseyr, $7.5 \mathrm{~km} \mathrm{~N}$ of & $16^{\circ} 28^{\prime} 54^{\prime \prime} \mathrm{N}, 16^{\circ} 27^{\prime} 42^{\prime \prime} \mathrm{W}$ \\
\hline$\overline{\text { Achram }}$ & $17^{\circ} 21.01^{\prime} \mathrm{N}, 12^{\circ} 23.98^{\prime} \mathrm{W}$ & & $19^{\circ} 11^{\prime} 13^{\prime \prime} \mathrm{N}, 16^{\circ} 19^{\prime} 25^{\prime \prime} \mathrm{W}$ & Muri & $16^{\circ} 26.265^{\prime} \mathrm{N}, 16^{\circ} 28.001^{\prime} \mathrm{W}$ \\
\hline Aleg & $17^{\circ} 3^{\prime} 12^{\prime \prime} \mathrm{N}, 13^{\circ} 54^{\prime} 58^{\prime \prime} \mathrm{W}$ & Agneïtir, Manate & $19^{\circ} 26^{\prime} 66^{\prime \prime} \mathrm{N}, 16^{\circ} 1$ & N'Diago, $20 \mathrm{Km}$ North o & of $16^{\circ} 26^{\prime} 16^{\prime \prime} \mathrm{N}, 16^{\circ} 28^{\prime} 00^{\prime \prime} \mathrm{W}$ \\
\hline Bogué & $16^{\circ} 35^{\prime} 36^{\prime \prime} \mathrm{N}, 14^{\circ} 16^{\prime} 28.8^{\prime \prime} \mathrm{W}$ & Akchâr & $19^{\circ} 55,059^{\prime} \mathrm{N}, 15^{\circ} 58,775^{\prime} \mathrm{W}$ & R'kiz & $16^{\circ} 55.110^{\prime} \mathrm{N}, 15^{\circ} 14.432^{\prime} \mathrm{W}$ \\
\hline & & Akjoujt & $19^{\circ} 44.636^{\prime} \mathrm{N}, 14^{\circ} 23.139 \mathrm{~W}$ & Rosso & $16^{\circ} 30.880^{\prime} \mathrm{N}, 15^{\circ} 48.784^{\prime} \mathrm{W}$ \\
\hline Dakhlet Nouâdhibou & & Azeffâl & $20^{\circ} 05^{\prime} \mathrm{N}, 15^{\circ} 47^{\prime} \mathrm{W}$ & Tamzakt & $17^{\circ} 27^{\prime} 48^{\prime \prime} \mathrm{N}, 16^{\circ} 09^{\prime} 00^{\prime \prime} \mathrm{W}$ \\
\hline$\overline{\text { Aghoueyyt and Inâl, be }}$ & tween & Bennichchâb & $19^{\circ} 27.996^{\prime} \mathrm{N}, 15^{\circ} 25.663^{\prime} \mathrm{W}$ & Tanit & $18^{\circ} 36^{\prime} 50^{\prime \prime} \mathrm{N}, 16^{\circ} 06^{\prime} 50^{\prime \prime} \mathrm{W}$ \\
\hline & $21^{\circ} 17.038^{\prime} \mathrm{N}, 15^{\circ} 08.371^{\prime} \mathrm{W}$ & Boudarga & $20^{\circ} 30.096^{\prime} \mathrm{N}, 14^{\circ} 53.541 \mathrm{~W}$ & Tiguent & $17^{\circ} 16^{\prime} 0.4^{\prime \prime} \mathrm{N}, 16^{\circ} 1^{\prime} 42.3^{\prime \prime} \mathrm{W}$ \\
\hline Baie de Cansado & $20^{\circ} 51^{\prime} 16.6^{\prime \prime} \mathrm{N}, 17^{\circ} 1{ }^{\prime} 32.8^{\prime \prime} \mathrm{W}$ & Châmi & $20^{\circ} 03.508^{\prime} \mathrm{N}, 15^{\circ} 52.915^{\prime} \mathrm{W}$ & Tiouilît & $18^{\circ} 52.311^{\prime} \mathrm{N}, 16^{\circ} 10.475^{\prime} \mathrm{W}$ \\
\hline
\end{tabular}

